\title{
La place du transfert d'énergie par pompage dans le parc de production $\mathbf{d}^{\prime} \mathbb{E} . \mathbf{D} . \mathbf{F}$. et l'aménagement de Grand'Maison
}

\author{
Share of energy transfer by pumping \\ in EDF's park of generating facilities \\ and development of Grand'Maison
}

\author{
M. Courier \\ Electricité de France \\ Chef adjoint des Services Etudes \\ Région d'Equipement Alpes-Lyon
}

\begin{abstract}
L'Aménagement de Grand'Maison, qu'EDF construit actuellement dans les Alpes dauphinoises, est une réalisation de taille exceptionnelle pour notre pays : avec ses $1800 \mathrm{MW}$ de puissance installée, Grand'Maison sera, à sa mise en service dans 3 ans, le plus important aménagement hydroélectrique français ${ }^{(1)}$.

C'est une opération mixte -à la fois gravitaire et de pompage- qui répond à un double objectif : créer un grand réservoir saisonnier accumulant des eaux d'été pour les turbiner en heures de forte demande d'hiver et créer une station de transfert d'énergie de forte puissance effectuant des cycles hebdomadaires de pompageturbinage, en hiver et en mi-saison.

Il nous a paru intéressant, avant de décrire les ouvrages de cet aménagement, de le situer dans l'ensemble du parc d'après 1985 et, en particulier, de vous présenter les données générales du transfert par pompage.

L'aspect technique, qui recouvre principalement les développements récents des groupes réversibles de grande puissance pour moyennes et hautes chutes, ayant été développé ce matin par M. de Maublanc, je centrerai mon propre exposé sur les aspects technicoéconomiques du transfert d'énergie.
\end{abstract}

\section{L'approche économique du transfert d'énergie}

\section{Les caractéristiques de la demande d'énergie électrique}

\section{L'évolution de la consommation annuelle}

De 1950 à 1973, la consommation française d'électricité a connu une croissance soutenue et assez régulière, à un taux moyen de $7,4 \% / \mathrm{an}^{(2)}$.

Après la stagnation $1974-75$, la demande a repris, à un rythme plus lent : 5,3\% en moyenne de 1973 à 1981 et la consommation intérieure (consommation nette + pertes) a atteint 258 TWh pour l'année 1981.
Les prévisions, pour la décennie à venir, se situent dans une fourchette de 315 à 320 TWh en 1985, 390 à 415 TWh en $1990^{(3)}$. Elles traduisent une augmentation de la consommation électrique d'environ $5 \%$ l'an, qui s'inscrit dans une croissance énergétique globale d'environ $2 \%$. L'électricité, qui permet d'arbitrer entre les différentes sources d'énergie primaire, verrait ainsi sa part dans le bilan énergétique passer de $30 \%$ actuellement à $40 \%$ en 1990 .

\section{Les variations de la demande}

La demande d'énergie électrique est sujette à des variations de deux ordres:

- des variations périodiques de grande amplitude ; - des variations aléatoires plus ou moins importantes et rapides.

Le producteur d'électricité est confronté à une difficulté particulière qui est d'adapter à tout instant l'offre à cette demande flucturante : l'électricité n'étant pas stockable - tout au moins directement et en quantité notable - l'équilibre de la production et de la consommation doit être réalisé, non pas en moyenne sur une période plus ou moins longue, mais en permanence et à une échelle de temps très fine. Le producteur doit donc disposer d'un parc d'équipement qui soit :

1) correctement ajusté, en volume global, à la demande maximale prévisible et

2) capable de performances dynamiques suffisantes.

Examinons plus en détail, pour préciser leur grandeur, les variations de la demande dont il est question.

(1) Le second en Europe, après l'Aménagement des Portes de Fer sur le Danube, si l'on excepte les grands Aménagements russes en Sibérie.

(2) Voisin du taux du doublement en 10 ans $(7,2 \%)$

(3) La figure 1 montre ce qu'aurait donné l'extrapolation de la tendance au doublement en 10 ans.

LA HOUILLE BLANCHE/N ${ }^{\circ}$ 5/6-1982 


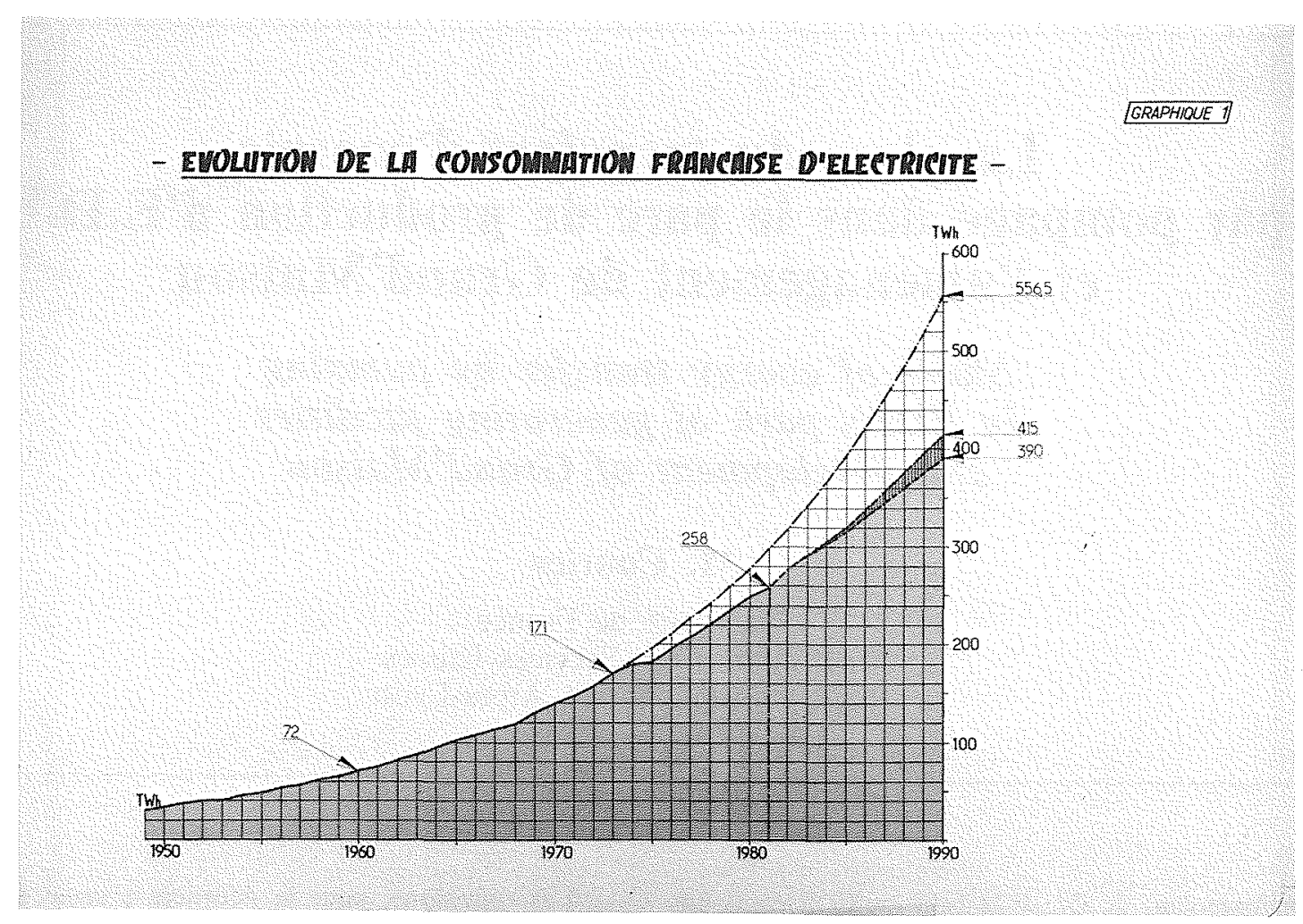

Figure 1 - Evolution de la consommation française d'électricité.

\section{Les variations périodiques}

Elles sont calquées sur les rythmes saisonnier, hebdomadaire et journalier, de l'activité humaine.

\section{Variations saisonnïeres}

Le tableau I montre le contraste entre les fortes consommations d'hiver et les creux de l'été, tels que constatés pour l'année 1980/81 et prévus pour l'année $1990 / 91$.

\section{Variations hebdomadaires et journalières}

Le tableau II et la figure 2 (courbes de charge types d'hiver et d'été) montrent :

- la baisse importante du niveau de consommation pendant les week-ends, par rapport aux jours ouvrables ; - la modulation journalière avec les pointes du matin et du soir, les creux de midi, de 18 heures et surtout de nuit.

\section{Les variations aléatoires}

Une observation fine de la demande met en évidence des variations aléatoires, plus ou moins rapides, d'amplitude parfois importante, induites par les fluctuations conjonctuelles de l'activité économique, culturelle, etc., ou par les conditions climatiques (température, luminosité...).

A titre d'exemple, la figure 3 donne une idée de la sensibilité de la demande pour un jour ouvrable d'hiver aux conditions de température, à l'horizon 1990 : une baisse de $5^{\circ} \mathrm{C}$ (sur l'ensemble du territoire) augmente la puissance appelée de $10 \mathrm{GW}$.

\section{Commentaires}

Plusieurs tendances, amorcées depuis quelques années, sont traduites dans les prévisions de consommation :

- accentuation nette du contraste saisonnier, en particulier du creux relatif de juillet-août ;

\begin{tabular}{|c|c|c|c|c|c|c|c|c|c|c|c|c|}
\hline \multicolumn{10}{|c|}{ Tableau I. - Variation saisonnière de la consommation intérieure } \\
en valeur réduite (consommation d'août =1,00) \\
\hline & $\mathrm{Jt}$ & $\mathrm{A}$ & $\mathrm{S}$ & $\mathrm{O}$ & $\mathrm{N}$ & $\mathrm{D}$ & $\mathrm{J}$ & $\mathrm{F}$ & $\mathrm{M}$ & $\mathrm{A}$ & $\mathrm{M}$ & $\mathrm{J}$ \\
\cline { 2 - 23 } \\
$\begin{array}{c}1980 / 81 \\
(1)\end{array}$ & 1,16 & 1,00 & 1,17 & 1,38 & 1,51 & 1,68 & 1,67 & 1,43 & 1,50 & 1,30 & 1,18 & 1,14 \\
\hline $\begin{array}{c}1990 / 91 \\
(2)\end{array}$ & 1,13 & 1,00 & 1,33 & 1,58 & 1,78 & 1,91 & 1,91 & 1,82 & 1,66 & 1,51 & 1,34 & 1,25 \\
\hline
\end{tabular}

(1) Consommations mensuelles réalisées, aux conditions climatiques effectives de 1980/81, sans correction de trend.

(2) Consommations mensuelles prévisionnelles, à température normale. 


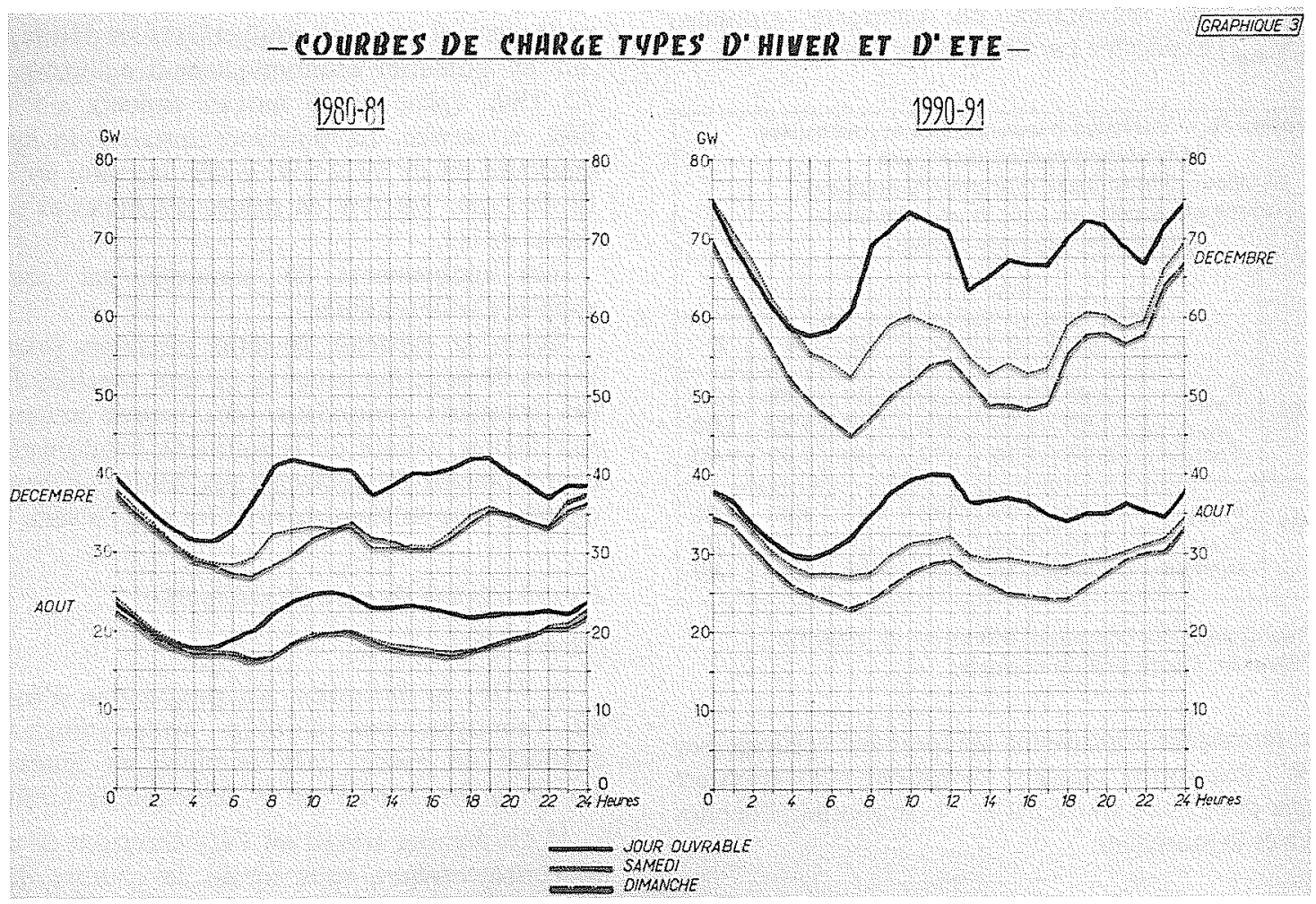

Figure 2 - Courbes de charge types d'hiver et d'été.

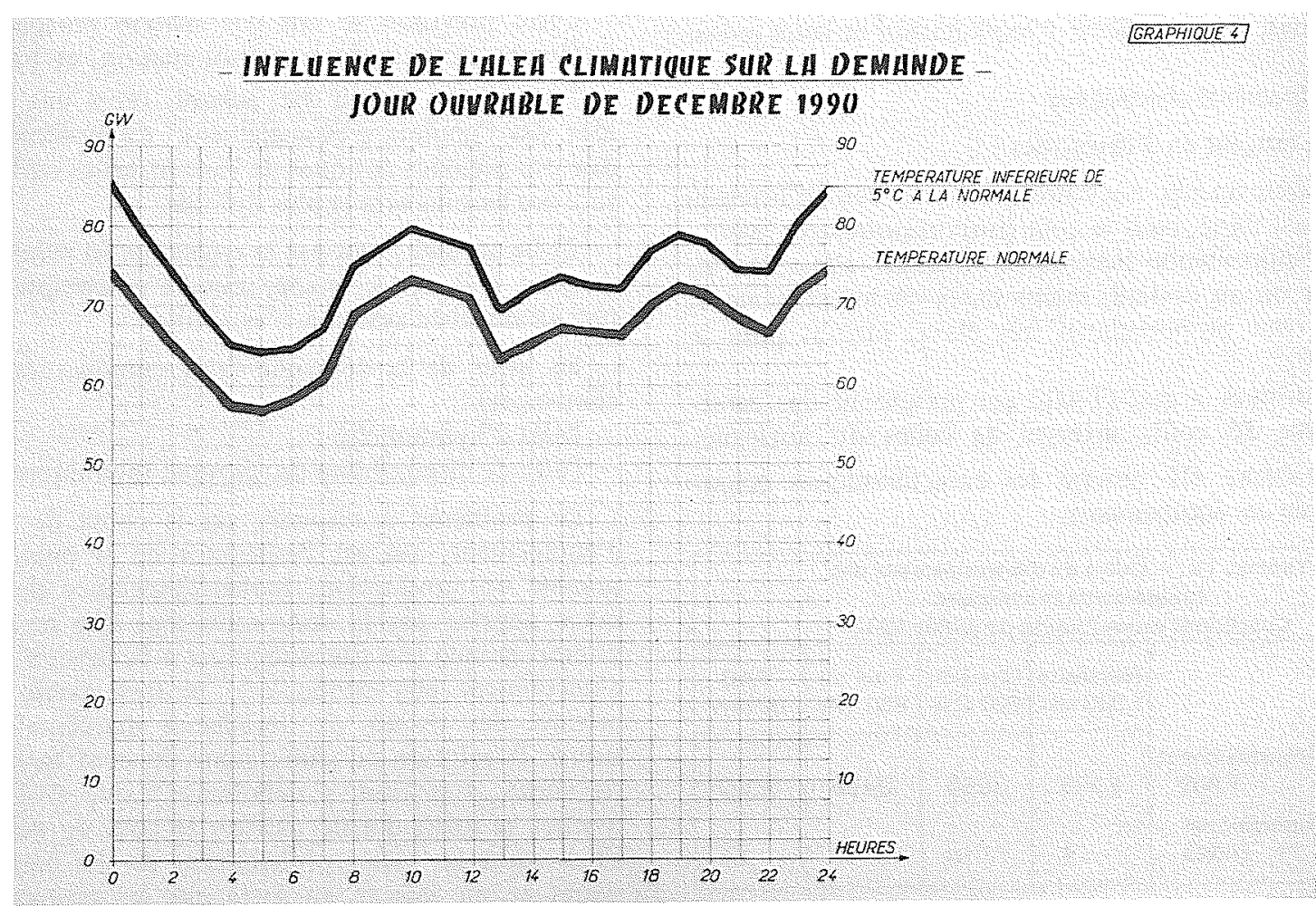

Figure 3 - Influence de l'aléa climatique sur la demande - Jour ouvrable de décembre 1990 
- légère accentuation du creux de week-end, sensible surtout le dimanche ;

- par contre, diminution du contraste journalier $\left(\mathrm{P}_{\max } / \mathrm{P}_{\min }\right)$ explicable par l'incitation tarifaire.

\begin{tabular}{|c|c|c|c|c|c|c|c|}
\hline \multicolumn{8}{|c|}{$\begin{array}{c}\text { Tableau II. - Variation hebdomadaire et journalière } \\
\text { de la consommation intérieure } \\
\text { en valeur réduite (base } 1,00 \text { pour la puissance } \\
\text { minimale appelée en août et pour l'énergie } \\
\text { journalièrè minimale servie). }\end{array}$} \\
\hline \multirow[b]{3}{*}{$\begin{array}{c}1980 / 81 \\
\text { (1) }\end{array}$} & \multirow[b]{3}{*}{$\begin{array}{l}P_{\max } \\
\mathrm{P}_{\min } \\
\text { Energie }\end{array}$} & \multicolumn{3}{|c|}{ Hiver } & \multicolumn{3}{|c|}{ Eté } \\
\hline & & $\mathrm{JO}$ & $\mathrm{S}$ & $D$ & JO & $S$ & $D$ \\
\hline & & $\begin{array}{l}2,62 \\
1,95 \\
2,06\end{array}$ & $\begin{array}{l}2,34 \\
1,75 \\
1,76\end{array}$ & $\begin{array}{l}2,32 \\
1,65 \\
1,72\end{array}$ & $\begin{array}{l}1,54 \\
1,11 \\
1,19\end{array}$ & $\begin{array}{l}1,48 \\
1,03 \\
1,02\end{array}$ & $\begin{array}{l}1,39 \\
1,00 \\
1,00\end{array}$ \\
\hline $\begin{array}{c}1990 / 91 \\
(2)\end{array}$ & $\begin{array}{l}\mathrm{P}_{\max } \\
\mathrm{P}_{\min } \\
\text { Energie }\end{array}$ & $\begin{array}{l}3,21 \\
2,48 \\
2,46\end{array}$ & $\begin{array}{l}3,05 \\
2,26 \\
2,15\end{array}$ & $\begin{array}{l}2,85 \\
1,93 \\
1,97\end{array}$ & $\begin{array}{l}1,73 \\
1,28 \\
1,30\end{array}$ & $\begin{array}{l}1,53 \\
1,16 \\
1,10\end{array}$ & $\begin{array}{l}1,45 \\
1,00 \\
1,00\end{array}$ \\
\hline
\end{tabular}

(1) Réalisation, aux conditions effectives de 1980/81.

(2) Prévision, à température normale.

Ces remarques fournissent un premier éclairage sur l'intérêt des trois grandes catégories de transfert d'énergie dont nous allons parler : le saisonnier, l'hebdo. madiare et le journalier.

\section{Les caractéristiques des équipements de production}

Pour satisfaire la demande, avec la meilleure qualité de service et dans les conditions les plus économiques, le producteur d'électricité dispose d'une panoplie d'équipements très diversifiés quant à leurs caractéristiques techniques, leur souplesse d'exploitation et leurs coûts.

\section{Les équipements thermiques}

Ils se rangent en 3 catégories :

- les équipements nucléaires, dont le coût d'investis. sement est élevé, mais le coût proportionnel très faible ; - les équipements thermiques fossiles, alimentés au charbon ou au fuel-oil, moins chers en investissement, mais à coût proportionnel très élevé, surtout pour le fuel-oil ;

- les turbines à gaz, (TAG) qui présentent des caractéristiques de coûts inverses de celles du nucléaire.

Le tableau III résume les caractéristiques économiques de ces équipements.

\begin{tabular}{|c|r|r|r|r|}
\hline \multicolumn{4}{|c|}{ Tableau III. - Coats de développement des } \\
equipements thermiques. \\
Conditions économiques de juillet 1981 \\
\hline & $\begin{array}{r}\text { Nuçéaire } \\
1300 \mathrm{MW}\end{array}$ & $\begin{array}{l}\text { Charbon } \\
600 \mathrm{MW}\end{array}$ & $\begin{array}{r}\text { Fuel oil } \\
600 \mathrm{MW}\end{array}$ & $\begin{array}{r}\text { TAG } \\
90 \mathrm{MW}\end{array}$ \\
\cline { 2 - 5 } & & & & \\
$\begin{array}{c}\text { Coût d'investissement } \\
\text { (1) F/kW }\end{array}$ \\
$\begin{array}{c}\text { Coût proportionnel } \\
\text { (2) c/kWh }\end{array}$ & 5200 & 3950 & 3400 & 2300 \\
\hline
\end{tabular}

(1) Pour une mise en service industriel en 1990.

(2) En 1990, avec hypothèses prospectives sur la dérive des coûts de combustible.

\section{Les équipements hydrauliques}

Les équipements hydrauliques gravitaires ne sont pratiquement plus des équipements en développement : sur un potentiel économiquement équipable d'environ $72 \mathrm{TWh}$, (productible moyen annuel), $64 \mathrm{TWh}$ sont déjà en service. La puissance installée en hydraulique gravitaire est de $17,9 \mathrm{GW}$, se décomposant en $7,9 \mathrm{GW}$ au fil de l'eau, 3,9 GW en usines d'éclusées et $6,1 \mathrm{GW}$ en usines de lac.

Les équipements de pompage, par contre, sont appelés à se développer.

Selon la capacité de leurs réservoirs (exprimée en heures de fonctionnement), ils sont capables d'effectuer des transferts journaliers (des heures creuses de nuit aux heures pleines de la journée), hebdomadaires (des heures creuses de week-end aux heures pleines des jours ouvrables) ou saisonniers (de l'été vers l'hiver).

\section{L'optimisation du parc de production}

\section{Méthodologie}

A EDF, la définition du programme d'équipement est l'aboutissement d'études assez complexes. J'en donnerai un aperçu très simplifié, en décrivant l'une des démarches suivies par la Direction de l'Equipement.

1) Le premier travail est l'établissement de prévisions de consommation, sous forme de courbes de charges aux différentes périodes de l'année, pour un certain nombre d'années repères. Les années particulièrement utiles à considérer sont les années $n+10$ et $n+15$, dans la mesure où le délai total d'études, de procédures et de réalisation d'un aménagement important est de 10 à 15 ans.

Cette prévision se fait par analyse prospective des différents secteurs de la consommation, et par région.

Lorsque ce travail est achevé, on a une première idée des "goulots d'étranglement" du système, c'est-àdire des périodes critiques de l'année, celles où la couverture des besoins sera la plus difficile.

2) Le second point est la recherche du volume global du parc. Il faut, à ce stade, prendre en compte le caractère aléa toire du système, c'est-à-dire :

- les aléas de la demande (aléa conjoncturel et aléa climatique) ;

- l'aléa d'hydraulicité ;

- l'aléa de disponibilité des groupes thermiques.

Le problème à résoudre est le choix de la marge d'équipement, qui est l'espérance de la puissance disponible en production, moins l'espérance de la puissance appelée par la consommation. Elle ne doit être ni trop grande (ne pas suréquiper à l'excès en prévision d'occurences peu probables), ni trop faible (ne pas prendre des risques de défaillance inacceptables). On trouve la solution en minimisant un coût total "investissement + gestion + défaillance" ou, si l'on veut tourner la difficulté de chiffrer le coût de défaillance, en dressant les bilans probabilistes sur les périodes critiques et en faisant en sorte que les probabilités de défaillance qui en découlent - et qui caractérisent la qualité de service - soient acceptables (comparables, pour fixer les idées, à ce qu'elles étaient dans le passé). 
3) En troisième lieu, on effectue par simulation le placement de l'hydraulique classique, au moyen de modèles de gestion sur monotones hebdomadaires (une monotone est une courbe de puissances moyennes horaires classées).

Il reste alors des courbes de charge, ou des monotones, à remplir avec des moyens thermiques.

4) Recherche de la consistance optimale du parc thermique.

La figure 4 permet de voir, sur une espérance de monotone annuelle de production thermique, comment l'on procède.

- En abscisses : les 8760 heures de l'année

- En ordonnées : le coût total de production des différents équipements (coût fixe $=$ investissement + charge actualisée d'exploitation, et coût proportionnel, représenté par la pente des demi-droites).

Les équipements sont empilés dans l'ordre des coûts proportionnels croissants : nucléaire, charbon, fuel-oil, turbine à gaz.
Le coût minimal de production est donné par l'enveloppe des demi-droites. En la rappelant sur la monotone, on obtient la position théorique des paliers d'équilibre.

Exemple :

$$
U_{N}^{c}=\frac{a_{N}-a_{c}}{b_{c}-b_{N}}
$$

avec :

$a_{N}, b_{N}=$ coût fixe et coût proportionnel du nucléaire.

$a_{e}, b_{c}=$ coût fixe et coût proportionnel du charbon. $U_{N}^{c}$ est la durée d'appel au charbon.

Avec les coûts $1990, U_{N}^{e}=4000$ à 4500 heures.

5) Enfin, la dernière étape consiste à analyser le disponible thermique $d$ 'heures creuses (principalement le disponible nucléaire et charbon) du parc ainsi défini et à rechercher son placement sous forme de transfert d'énergie. Cela n'est possible qu'avec des modèles plus fins que les précédents et nous y reviendrons plus loin.

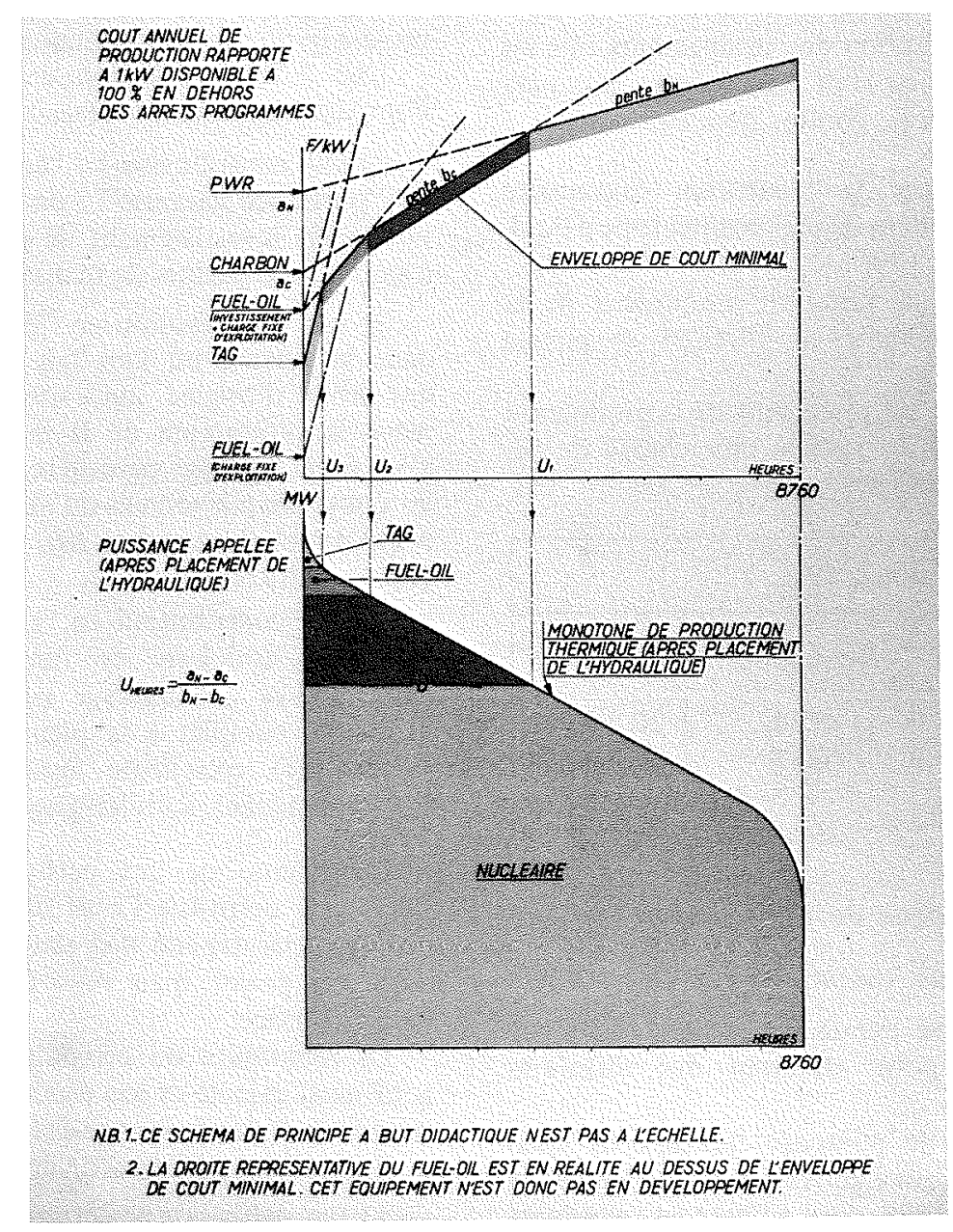

Figure 4 - Recherche de l'optimum du parc de production. Equilibre entre équipements en développement - Schéma de principe. 


\section{Consistance du parc de production 1990}

La figure 5 montre les monotones annuelles 1980 , 1985 et 1990 .

En 1980, les effets de la crise ne sont pas encore compensés et le parc est loin d'être optimal. Il est encore très marqué par la part importante du thermique à flamme. Le nucléaire n'occupe qu'une partie de la base du diagramme de charge. Les coûts marginaux de production sont donc entièrement tributaires des combustibles fossiles.

C'est en principe vers 1984 que le nucléaire sortira de la base. Il représentera alors environ $50 \%$ de la production électrique.

En 1990, le parc est réoptimisé. La production nucléaire représente un peu plus de $70 \%$ du total. La durée d'appel au charbon (durée d'équilibre à l'interférence nucléaire/charbon) s'établit entre 4000 et 4500 heures, c'est-à-dire à la moitié de l'année. Le nucléaire est donc marginal pendant la moitié du temps. Mais la répartition dans l'année des heures à nucléaire marginal est très inégale : $90 \%$ du temps en été, $50 \%$ en demisaison et seulement $20 \%$ en hiver (pendant les weekends). Ces caractéristiques du disponible nucléaire sont une donnée essentielle de l'étude économique du transfert d'énergie.

On voit que la contribution des équipements fuel est devenue très faible : ils ne sont plus appelés que quelques heures par jour, à la pointe, pendant environ 3 mois d'hiver.

Le tableau IV donne la répartition saisonnière moyenne du temps pendant lequel chaque type d'équipement sera marginal, en 1990.

$\mathrm{La}$ figure 6 montre la variation saisonnière de la production, telle que réalisée en $1980 / 81$ et prévue pour 1990/91.

La figure 7 s'intéresse au placement de la production sur la courbe de charge du jour le plus chargé de décembre 1981 et décembre 1990.

\begin{tabular}{|l|c|c|c|}
\hline \multicolumn{4}{|c|}{ Tableau IV } \\
\hline & Hiver & Demi-saison & Eté \\
\cline { 2 - 4 } Nucléaire & $20 \%$ & $55 \%$ & 90 à $95 \%$ \\
Charbon & $50 \%$ & $40 \%$ & 5 à $10 \%$ \\
Fuel Oil & $30 \%$ & $5 \%$ & 0 \\
\hline
\end{tabular}

\section{La justification économique du transfert par pompage}

Le pompage hydraulique comme moyen de transfert

Le pompage est utilisé depuis longtemps, en France comme à l'étranger, pour effectuer des transferts d'énergie.

La première station de pompage française de puissance significative, celle du Lac Noir dans les Vosges, a été mise en service en 1938 pour utiliser les excédents d'heures creuses de la centrale au fil de l'eau de Kembs. A la même époque, les Allemands associaient du pompage aux centrales thermiques de la Ruhr.

Mais le pompage hydraulique n'est pas le seul moyen de transfert et EDF s'est intéressé aux techniques concur- rentes : accumulation thermique dans un fluide, production d'hydrogène par électrolyse, accumulation électrochimique, air comprimé, volants inertiels, etc.

Certains de ces procédés, sur lesquels on avait fondé des espoirs, n'ont pas tenu leurs promesses. Les seules alternatives un peu crédibles à moyen terme sont l'air comprimé et les batteries d'accumulateurs. A l'heure actuelle et même en tenant compte des prévisions les plus optimistes sur l'amélioration des rendements et des coûts, aucun d'eux n'est compétitif avec une installation de pompage réalisée sur un bon site.

Dans les installations de pompage en construction ou en projet, le rendement du cycle est de 0,72 à 0,75 , c'est-à-dire qu'il faut consommer $1,4 \mathrm{kWh}$ en pompage pour produire $1 \mathrm{kWh}$ en turbinage.

\section{La méthodologie des études de rentabilité du pompage}

Il faut d'abord noter que l'étude économique du pompage est d'une grande complexité ; elle nécessite l'examen "à la loupe" de la gestion de l'ensemble du parc et donc l'utilisation de modèles de simulation à pas de temps très fin (Placement sur courbes de charge de préférence ou à défaut sur monotone journalière). A EDF, ce type d'étude n'a pas pu être décentralisé, comme l'avaient été les études de rentabilité des projets hydrauliques gravitaires.

Une S.T.E.P. (Station de Transport d'Energie par Pompage) apporte au réseau une triple contribution :

1) une contribution en "économie de défaillance" ou en "puissance garantie" ; on peut la chiffrer par l'économie en investissement de moyen de pointe (turbine à gaz) qu'elle permet de réaliser.

2) une contribution en "économie de combustible" dans la mesure où l'on fait appel pour le pompage à des $\mathrm{kWh}$ d'origine nucléaire ou charbon et où l'on place la production de la S.T.E.P. en substitution de $\mathrm{kWh}$ fuel. La crise pétrolière, qui a creusé l'écart entre les coûts proportionnels du nucléaire et du fuel, (Fig. 8), a évidemment accentué très fortement la valeur d'une telle substitution.

3) une contribution en "avantages cinétiques" liée à la grande souplesse de fonctionnement des groupes hydrauliques : démarrage rapide (Grand'Maison passe de l'arrêt complet à la pleine puissance en moins de 2 minutes), excellente aptitude au suivi de charge et, en particulier, au téléréglage.

L'ensemble de ces performances, convenablement valorisées, constitue l'actif de la S.T.E.P. Il faut noter que cet actif dépend non seulement de la qualité intrinsèque du projet, mais aussi de la quantité de pompage déjà en service.

La rentabilité du projet se juge en comparant son actif ainsi calculé et son passif, somme des coûts d'investissement et des frais actualisés d'exploitation.

En pratique, on procède de la façon suivante:

1) Les différents projets en catalogue sont dimensionnés séparément et classés par ordre de valeur intrinsèque décroissante.

2) A l'aide du modèle de gestion, on recherche les actifs annuels successifs du projet que l'on veut ajouter au parc de pompage déjà décidé. 


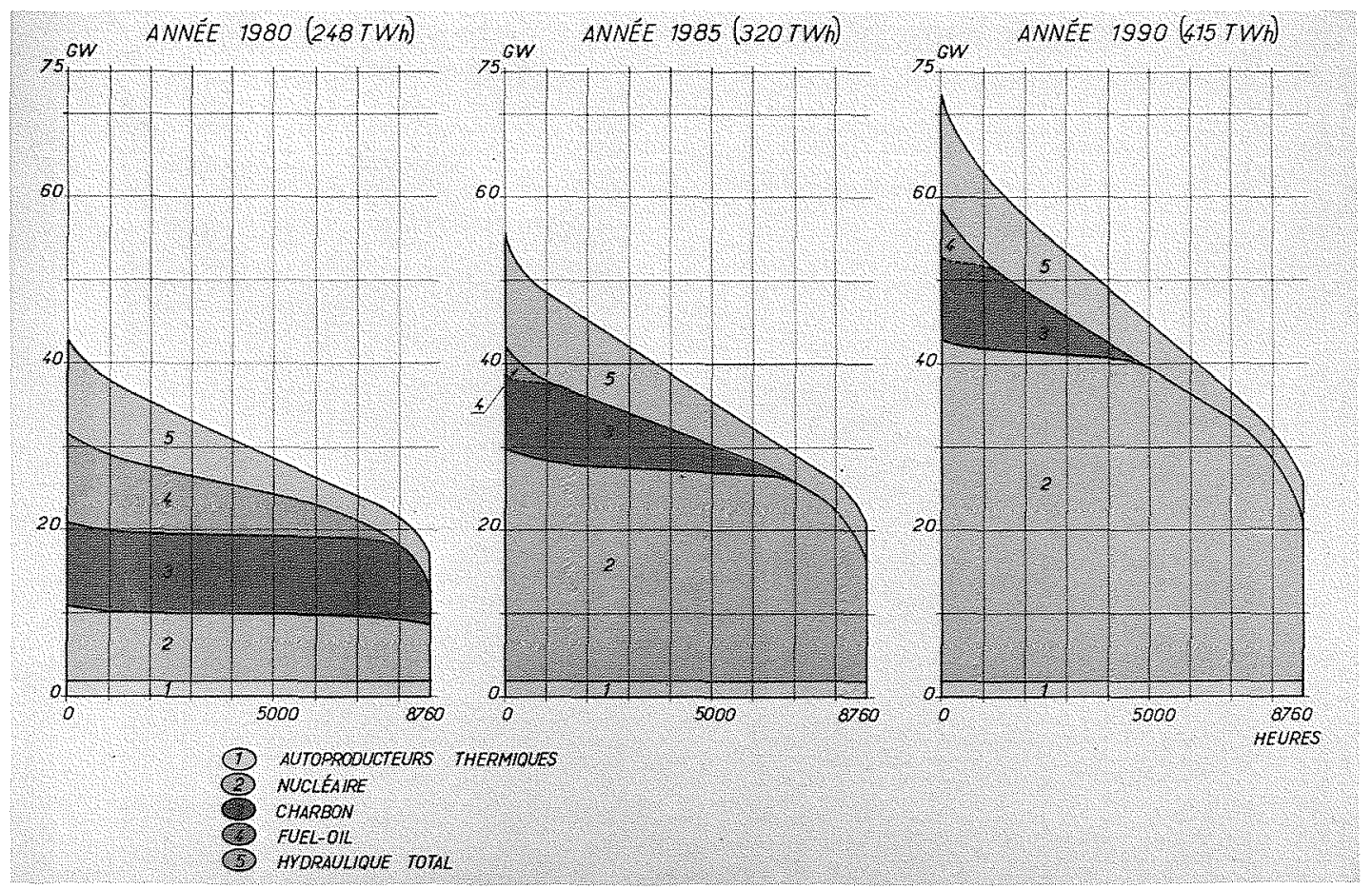

Figure 5 - Monotones annuelles de production et de consommation.

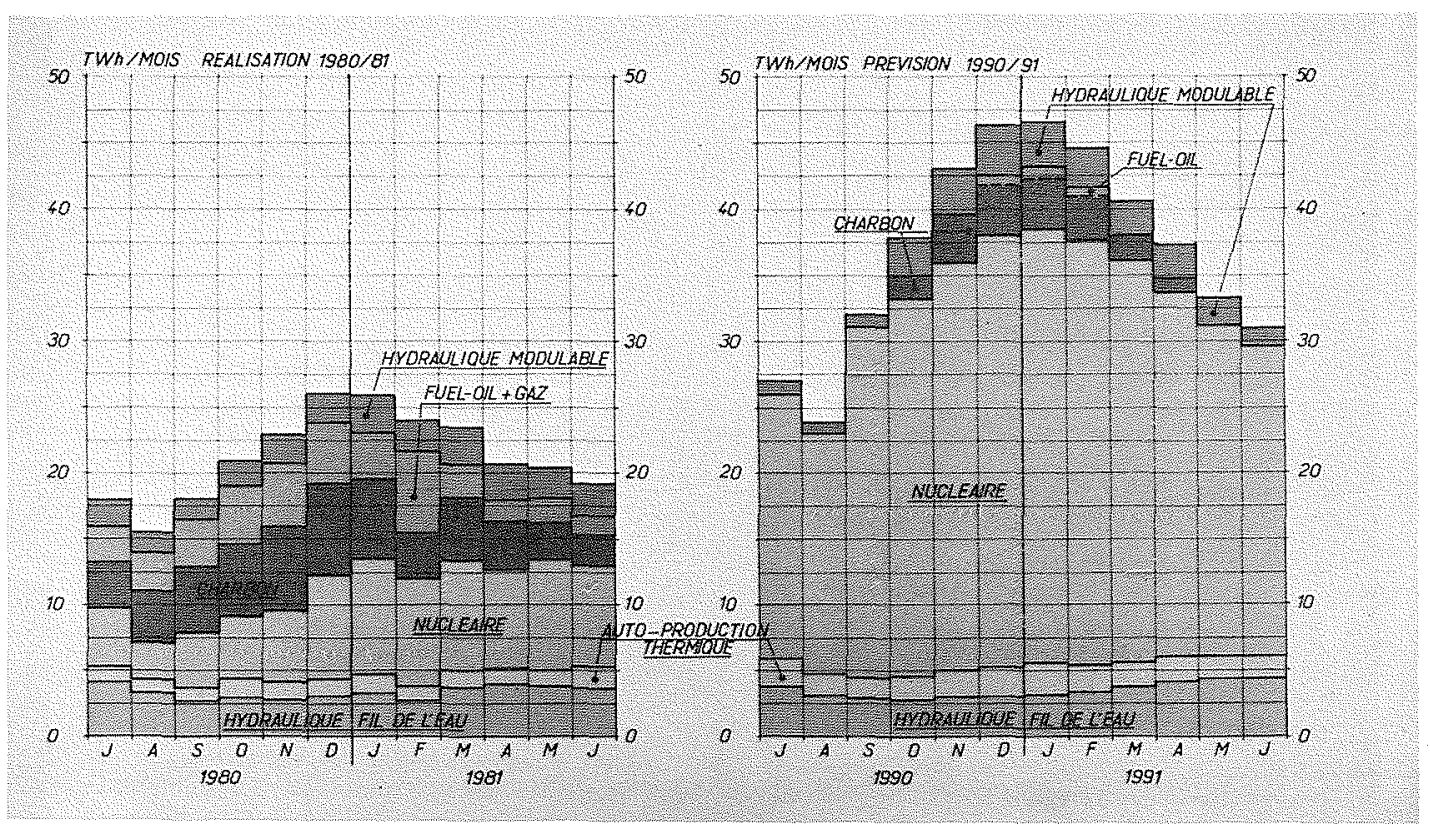

Figure 6 - Variation saisonnière de la production. 

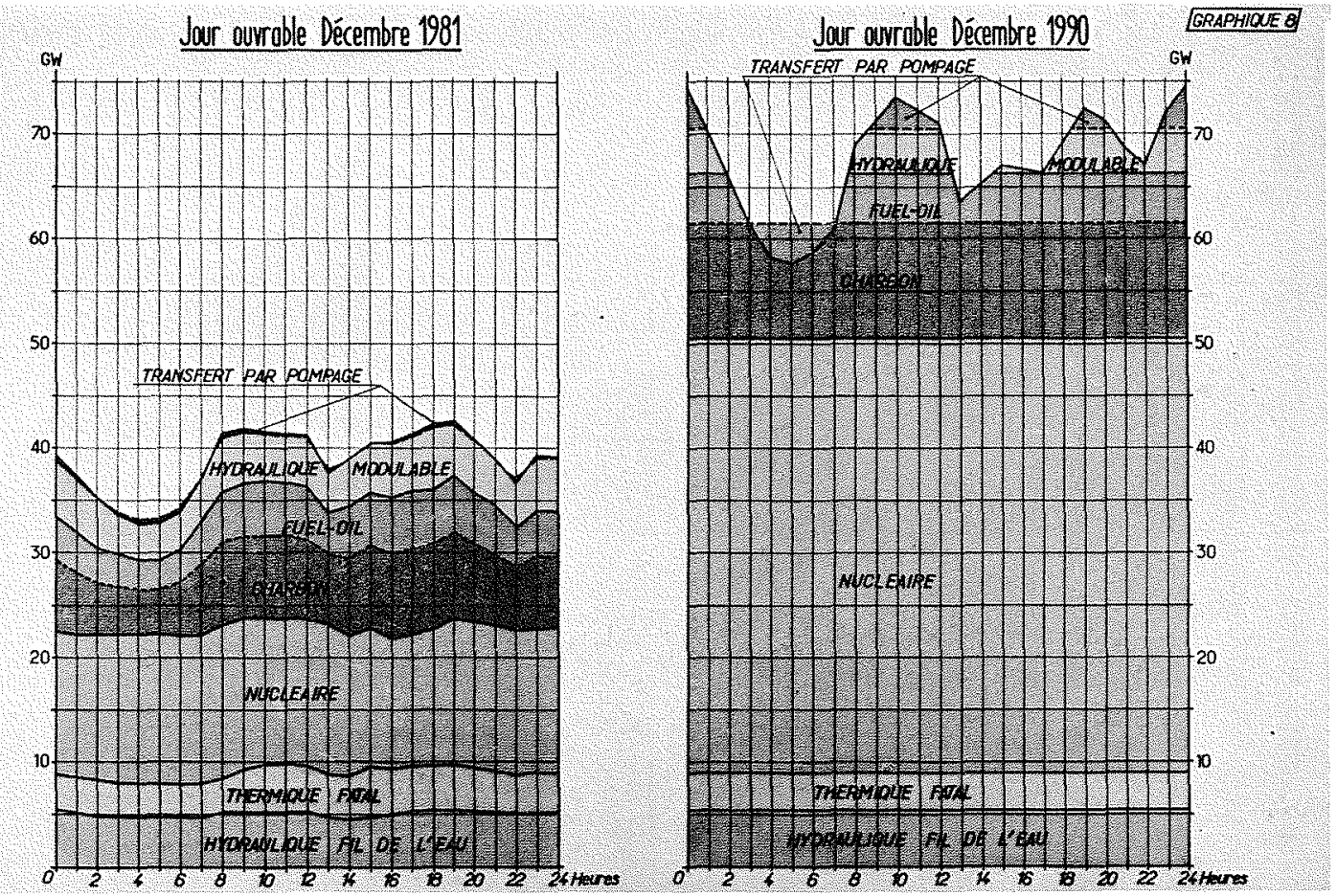

Figure 7 - Placement de la production sur la courbe de charge du jour le plus chargé.

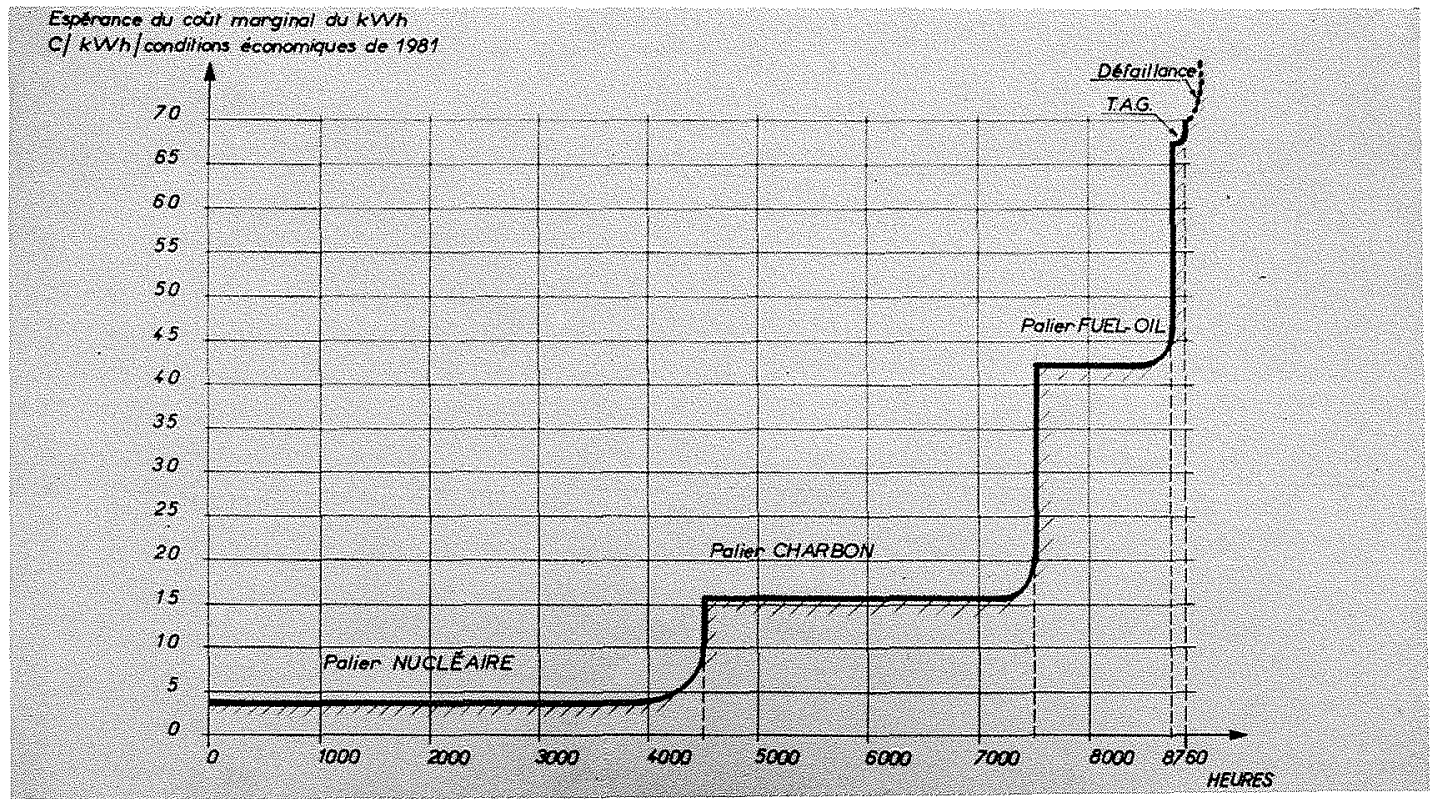

Figure 8 - Coût marginal de production en fonction de la durée pendant laquelle chaque équipement est marginal. (Prévision pour l'année 1990). 
3) En comparant la série croissante des actifs annuels successifs et le coût d'anticipation du projet, on trouve l'année à partir de laquelle il devient rentable.

\section{La valeur économique des différentes catégories de pom- page}

L'étude économique confirme ce que laissait prévoir l'analyse du disponible d'heures creuses et sa répartition dans l'année. Elle conduit à classer les différentes catégories de pompage, par intérêt décroissant, dans l'ordre suivant :

1) Le pompage à réserve saisonnière - exemple : Grand'Maison - C'est de loin le plus intéressant, car il permet :

- d'opérer les transferts de l'été et de la mi-saison vers l'hiver qui sont les plus valorisants en économie de combustible. De ce point de vue, le transfert par pompage saisonnier n'est pas très différent des transferts saisonniers gravitaires réalisés par les usines classiques de lac ;

- de diminuer le risque de défaillance en hiver beaucoup plus que ne peut le faire le pompage purement hebdomadaire car ce dernier est beaucoup plus sensible aux aléas (notamment à l'aléa climatique).

- d'opérer les transferts hebdomaires permettant à la fois d'économiser le fuel et le charbon en hiver, le charbon en demi-saison, et de diminuer le risque de défaillance.

2) Le pompage hebdomadaire associe à un lac existant - exemple : Super Bissorte.

Ce type de pompage présente partiellement les avantages du précédent.

3) Le pompage à réserve purement hebdomaire exemple : Montézic.

Il ne permet d'opérer que des transferts hebdomadaires, qui se justifient en hiver et en demi-saison ; mais ces transferts suffisent pour assurer la rentabilité des meilleurs projets de cette catégorie.

4) Le pompage à réserve purement journalière.

Il ne permet d'opérer que des transferts à l'échelle de la journée ; ce type de pompage est fortement tributaire des aléas qui conditionnent les disponibilités en heures creuses des jours ouvrables d'hiver. Il faut d'ailleurs observer que ces disponibilités sont déjà exploitées par les installations saisonnières et hebdomadaires; la contribution du pompage purement journalier à la diminution du risque de défaillance est donc très faible.

On notera toutefois que des installations de pompage journalier peuvent trouver leur justification dans le cas particulier de régions excentrées sur le réseau de grand transport et dépourvues de moyens propres de modulation.

\section{Le programme de pompage $D^{\prime} E D F$}

En plus de la station du "Lac Blanc - Lac Noir" déjà citée, qui fonctionne depuis 1938, EDF a mis en service, dans la période 1973-1978, les S.T.E.P.de:

- Vouglans 2

$50 \mathrm{MW}$

- Revin
- Ste-Croix 2

$50 \mathrm{MW}$, - La Coche $320 \mathrm{MW}$, - Arc-Isère

$480 \mathrm{MW}$.

La puissance totale de pompage en service au 31.12.81 s'élève à $1,8 \mathrm{GW}$.

Le programme en cours de réalisation est mentionné dans le tableau. $V$.

\begin{tabular}{|c|c|c|c|c|}
\hline \multicolumn{5}{|c|}{ Tableau V } \\
\hline $\begin{array}{l}\text { Année } \\
\text { de } \\
\text { programme }\end{array}$ & Opérations & Type & $\begin{array}{c}\text { Puissance } \\
\text { installeee } \\
\text { MW }\end{array}$ & $\begin{array}{l}\text { Mise } \\
\text { en } \\
\text { service }\end{array}$ \\
\hline 1976 & Montézic & hebdo & 920 & 1982 \\
\hline 1978 & Le Truel & hebdo & 38 & 1982 \\
\hline 1978 & $\begin{array}{l}\text { Grand' } \\
\text { Maison }\end{array}$ & $\begin{array}{l}\text { saisonnier } \\
+ \text { gravitaire }\end{array}$ & $1200(1)$ & 1985 \\
\hline 1979 & $\begin{array}{l}\text { Super } \\
\text { Bissorte }\end{array}$ & $\begin{aligned} & \text { hebdo } \\
+ & \text { gravitaire }\end{aligned}$ & $600(2)$ & 1986 \\
\hline
\end{tabular}

(1) Plus $600 \mathrm{MW}$ Pelton.

(2) Plus 150 MW Pelton.

L'orientation des études pour les opérations futures est la suivante :

\section{En ce qui concerne le pompage saisonnier}

Le gisement est relativement limité. Les cuvettes saisonnières les plus intéressantes (celles qui donnaient le plus grand nombre de $\mathrm{m}^{3}$ d'eau stockés par $\mathrm{m}^{3}$ de barrage et qui étaient à peu près exemptes d'aléas géologiques) sont déjà aménagées. Les sites nouveaux présentent généralement, soit une topographie moins favorable, soit des difficultés géologiques, soit des difficultés d'environnement (et assez fréquemment les trois à la fois).

Deux opérations saisonnières importantes, l'Aménagement du Clou en Haute-Isère et l'Aménagement de la Haute-Romanche, sont actuellement à l'état d'avantprojet sommaire et pourraient être engagées vers 198687 , pour être mises en service peu après 1990.

En 1990, et au-delà, le pompage saisonnier n'utilisera finalement qu'une partie du disponible nucléaire d'été. Il pourrait donc y avoir place, à terme, pour d'autres procédés de transfert d'énergie saisonnier.

\section{En ce qui concerne le pompage hebdomadiare}

Son développement sera nécessairement progressif et adapté à l'accroissement du disponible d'heures creuses pendant les week-ends d'hiver.

Les sites susceptibles de recevoir un équipement de pompage hebdomadaire à des coùts avantageux existent en assez grand nombre, principalement dans la moitié Sud du pays. L'objectif d'EDF est de les répertorier, d'identifier pour chacun d'eux les aléas techniques et les difficultés d'environnement au sens large, de sélectionner les meilleurs d'entre eux et d'étudier plus en détail les quelques opérations qui pourraient être mises en service entre 1990 et l'an 2000. 


\section{L'aménagement de Grand'Maison}

\section{L'historique du projet}

Les premières études de la chute de Grand'Maison remontent aux années 1950 . La cuvette glaciaire de Grand'Maison dans la haute vallée de l'Eau d'Olle avait retenu l'attention des projeteurs d'EDF en quête de grandes retenues d'altitude. La proximité de l'Isère permettait d'envisager une chute directe de $1400 \mathrm{~m}$. Les premiers projets fondés sur une utilisation purement gravitaire des eaux prévoyaient, pour remplir le réservoir de Grand'Maison, un très long réseau d'adductions souterraines dérivant les affluents de rive droite de la Romanche et les affuents de rive gauche de I'Arc jusque sous le Col du Galibier, et captant également les torrents du massif d'Allevard.

$\mathrm{Au}$ fur et à mesure de l'avancement des études, il apparut :

- qu'une grande partie des galeries d'adduction amont aurait à traverser des terrains difficiles, en particulier dans le massif des Grandes Rousses ;

- que le poids croissant des contraintes d'environnement rendait administrativement très difficile le détournement des bassins versants en question ;

- et surtout que l'évolution du parc de production, avec la diminution de la part relative de l'hydraulique classique, la place faite au pompage, l'apparition de besoins importants en téléréglage, justifiaient de nouveaux critères de conception et de dimensionnement des projets de ce type.

Finalement, les adductions gravitaires amont furent abandónnées progressivement au profit d'un pompage d'été et la chute directe sur l'Isère fut remplacée par une chute de $950 \mathrm{~m}$ suivant le cours de l'Eau d'Olle. En série avec cette chute, il est prévu, pour une réalisation ultérieure, une chute de $450 \mathrm{~m}$, dite RomancheIsère, entre la Romanche au droit de Livet, et l'Isère à hauteur du Versoud, à $15 \mathrm{~km}$ à l'amont de Grenoble.

\section{Les caractéristiques principales de l'aménagement}

\section{Hydrologie}

La superficie du bassin versant est de $50 \mathrm{~km}^{2}$ au barrage de Grand'Maison et de $115 \mathrm{~km}^{2}$ au barrage du Verney (réservoir inférieur). Les apports moyens utilisables pour le remplissage saisonnier (compte tenu des débits réservés) s'élèvent à $105 \mathrm{Mm}^{3}$ à Grand'Maison et à $78 \mathrm{Mm}^{3}$ au Verney.

\section{Hauteurs de chute}

Niveaux du réservoir supérieur $=1695 \quad$ à $1590 \mathrm{NGF}$ Niveaux du réservoir inférieur $=768,50$ à $740 \mathrm{NGF}$

Chute brute

pour les groupes réversibles $=955$ à $821,50 \mathrm{~m}$ pour les groupes Pelton $\quad=922,40$ à $817,40 \mathrm{~m}$

\section{Débits}

Maximal en turbinage (12 groupes) $=217 \mathrm{~m}^{3} / \mathrm{s}$ Maximal en pompage ( 8 groupes) $=135 \mathrm{~m}^{3} / \mathrm{s}$

\section{Puissances}

Totale installée (12 groupes) $=1800 \mathrm{MW}$

Maximale en turbinage (12 groupes) $=1680 \mathrm{MW}$

Maximale en pompage ( 8 groupes $)=1275 \mathrm{MW}$

\section{Les ouvrages de l'aménagement}

\section{Présentation générale (Fig. 9)}

L'aménagement se situe à $30 \mathrm{~km}$ de Grenoble, dans la vallée de l'Eau d'Olle, affluent de rive droite de la Romanche. L'eau d'Olle prend sa source sous le Col de la Croix de Fer et s'écoule entre les Chaînes de Belledonne et des Grandes Rousses, pour rejoindre la Romanche à l'aval de la plaine de Bourg-d'Oisans (Fig. 10).

Les massifs cristallins de Belledonne et des Grandes Rousses sont séparés par une dépression liasique la vallée de Vaujany. Le cours supérieur et le cours inférieur de l'Eau d'Olle se développent dans l'axe de cette dépression ; le cours médian décrit un large coude dans le flanc Est du massif de Belledonne.

Plus précisément, dans le secteur de l'Aménagement, le cristallin de Belledonne est constitué par des gneiss, amphibolites et granits et le lias par de marno-calcaires et schistes ardoisiers.

Le projet comporte (Fig. 11) :

- un réservoir supérieur, la retenue de Grand'Maison, d'une capacité utile de $132 \mathrm{Mm}^{3}$;

- un réservoir inférieur, le bassin du Venery, d'une capacité utile de $14 \mathrm{Mm}^{3}$;

- une usine réversible de $1200 \mathrm{MW}$ de puissance installée,

- une usine Pelton de $600 \mathrm{MW}$.

\section{Le barrage de Grand'Maison}

Il permet de créer, à la cote 1695 , une retenue dont le remplissage est assuré par les apports naturels et par pompage des hautes eaux de printemps et d'été dans le bassin inférieur du Verney.

Le site retenu pour l'implantation du barrage présente une morphologie de verrou glaciaire avec un remplissage alluvial de 10 à $50 \mathrm{~m}$ d'épaisseur et un important éboulis de pente en rive droite. Il est traversé en diagonale par un contact géologique lias sur cristallin qui a nécessité une attention particulic̀re, mais qui s'est révélé parfaitement franc et serré.

L'ouvrage adopté est une digue en remblai à noyau vertical épais fondé au rocher, recharge aval en terre caillouteuse, recharge amont en enrochement (Fig. 12). Ses caractéristiques géométriques sont les suivantes :

- Hauteur au-dessus du terrain naturel $=140 \mathrm{~m}$

- Hauteur maximale sur fondations $=160 \mathrm{~m}$

- Longueur en crête $\quad=550 \mathrm{~m}$

- Largeur en crête $\quad=10 \mathrm{~m}$

- Pente moyenne du talus amont $\quad=1 / 1,7$

- Pente moyenne du talus aval $=1 / 2,1$

- Volume du noyau $=1,9 \mathrm{hm}^{3}$

- Volume d'enrochements $=4,7 \mathrm{hm}^{3}$

- Volume total du remblai $=12,5 \mathrm{hm}^{3}$

Le matériau constitutif du noyau est une terre liasique à granulométrie étalée, prélevée dans la cuvette à 


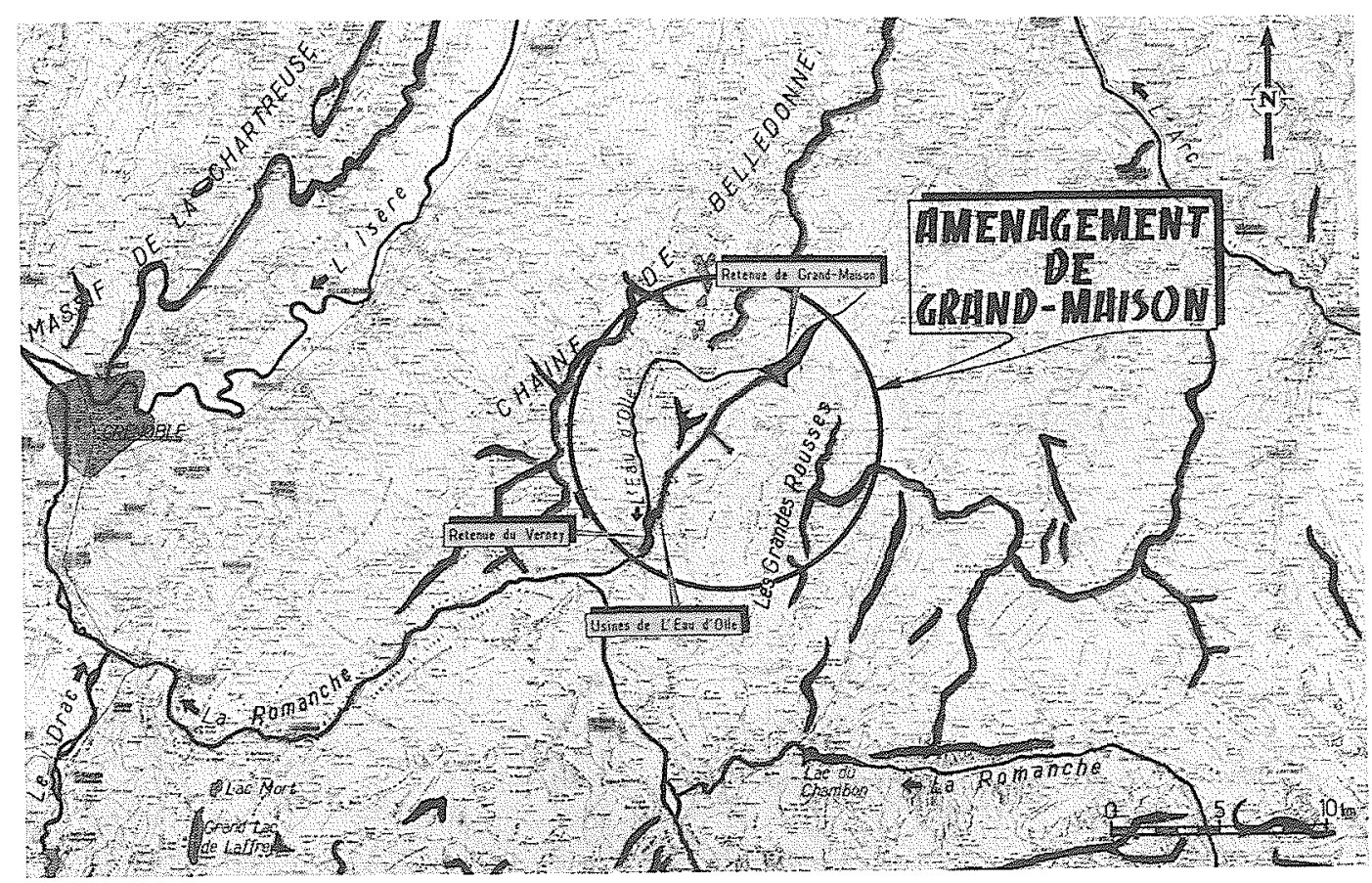

Figure 9 - Aménagement de Grand'Maison. - Plan de situation.

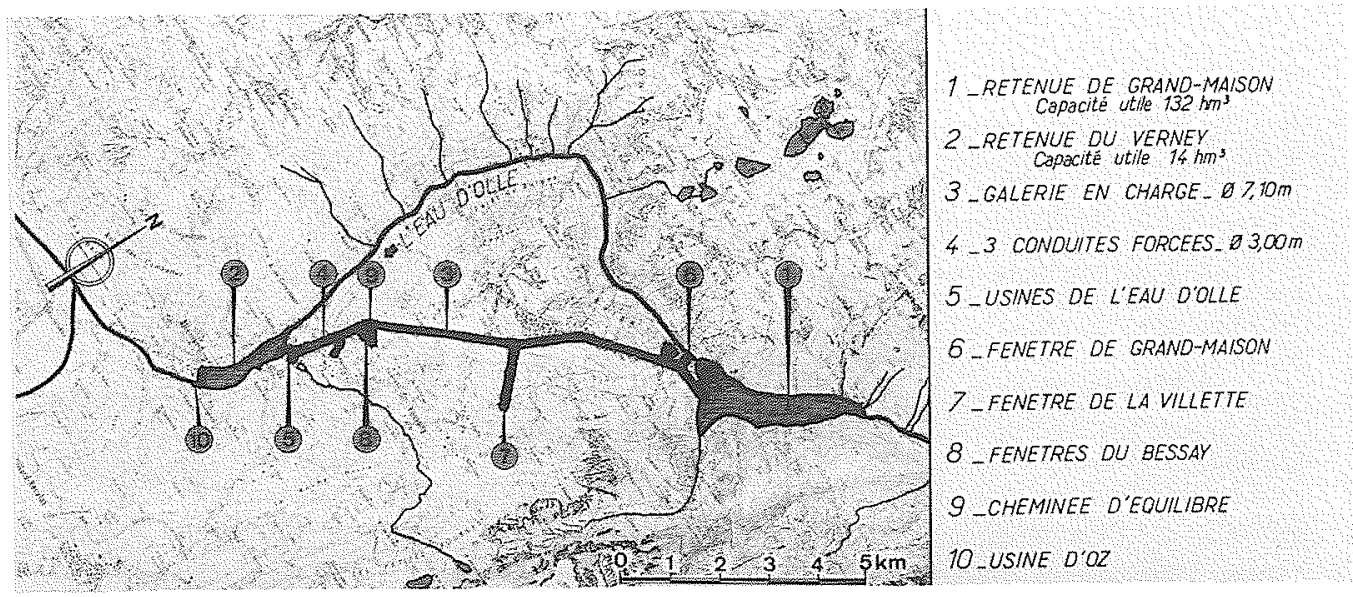

Figure 10 - Aménagement de Grand'Maison - Plan d'ensemble.

proximité du barrage, donnant après compactage des perméabilités comprises entre $10^{-7}$ et $10^{-8} \mathrm{~m} / \mathrm{s}$.

Les terres caillouteuses semi-perméables qui constituent l'essentiel de la recharge aval et une partie de la recharge amont proviennent d'un gite dans la cuvette, en rive droite de l'Eau d'Olle.

Les enrochements de la recharge amont et du rip rap sont extraits d'une carrière ouverte $500 \mathrm{~m}$ à l'aval du barrage, dans les gneiss de la rive gauche. Les filtres et drains sont obtenus par concassage et criblage des enrochements de la carrière.
L'exutoire du drain à l'aval du noyau se fait gravitairement vers la galerie de contrôle périmétrale raccordée à une galerie sous-fluviale débouchant à l'aval dans la gorge de l'Eau d'Olle.

Les ouvrages de contrôle des débits comportent : - une vidange de fond aménagée en galerie dans la rive gauche avec vanne plate de garde et vanne segment de réglage ;

- un évacuateur de crue avec seuil à surface libre, puits incliné de $60 \mathrm{~m}$, galerie de $200 \mathrm{~m}$, coursier extérieur et cuillère de dissipation. 


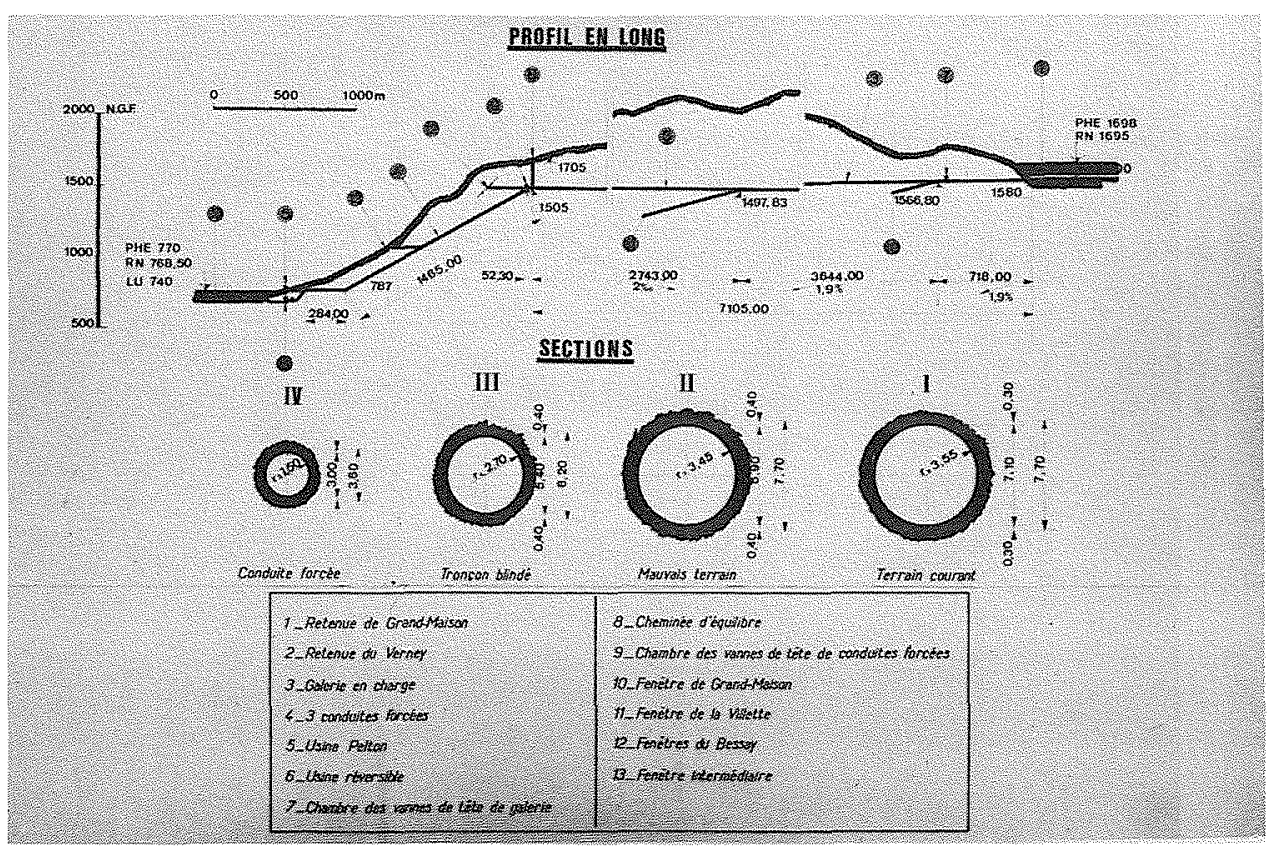

Figure 11 - Aménagement de Grand 'Maison. - Profil en long.

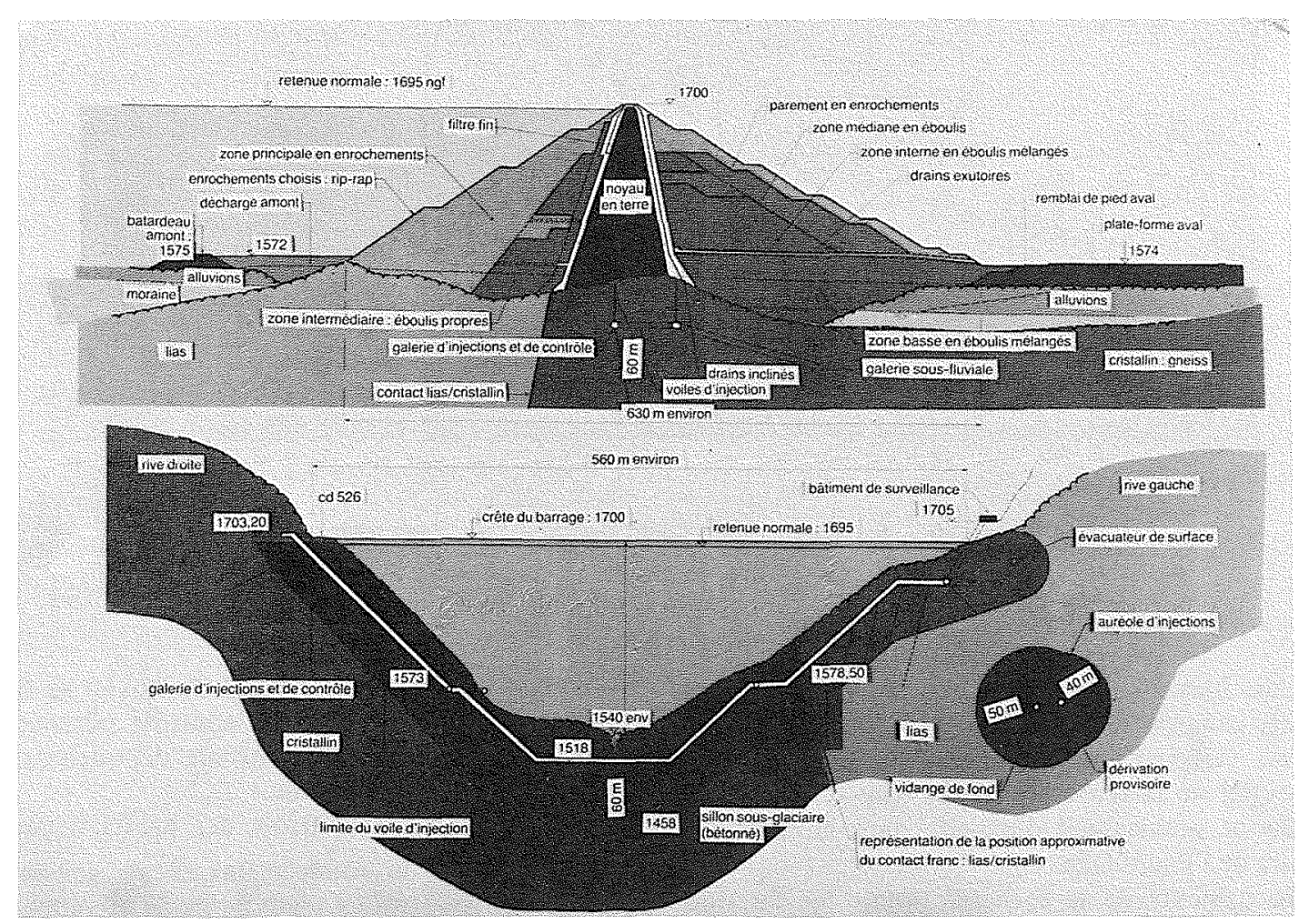

Figure 12 - Barrage de Grand 'Maison - Coupes transversale et longitudinale. 


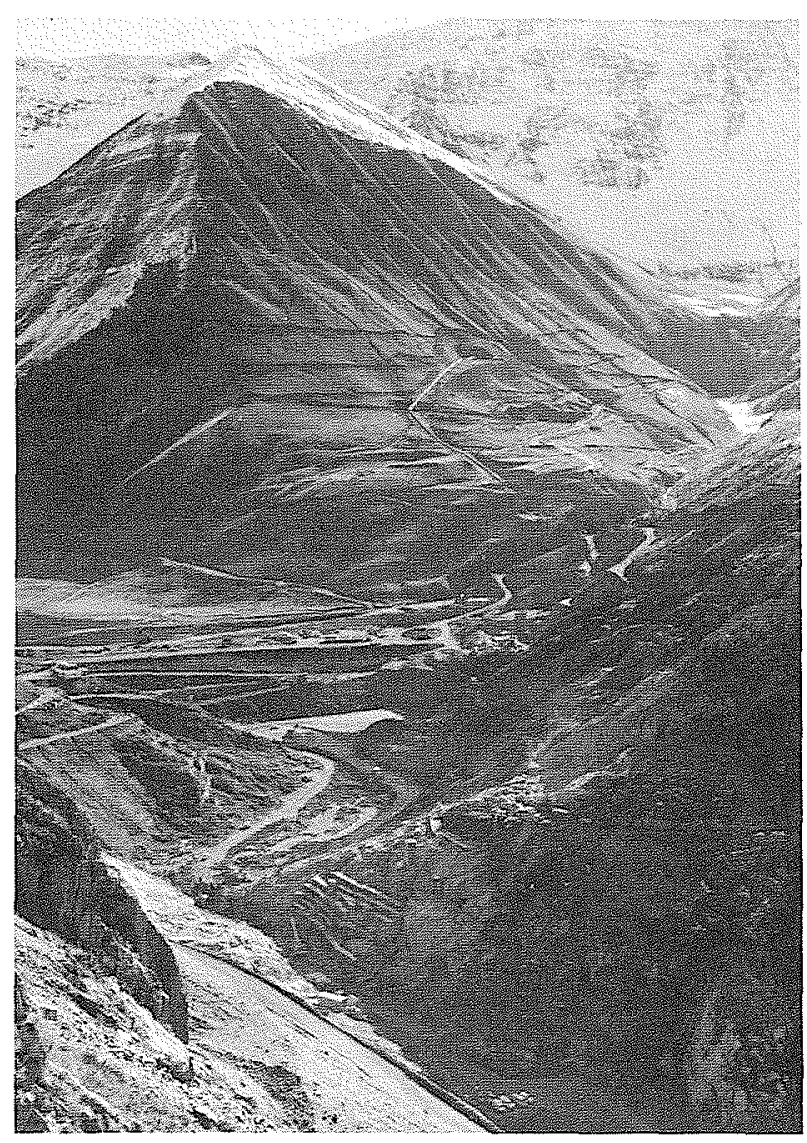

Figure 13 - Site du barrage de Grand'Maison, vu de l'aval. - au premier plan - échafaudages pour les injections du rocher. - à l'arrière-plan - gîte des terres à noyau, avec le tapis transporteur (photo MIRABEL).

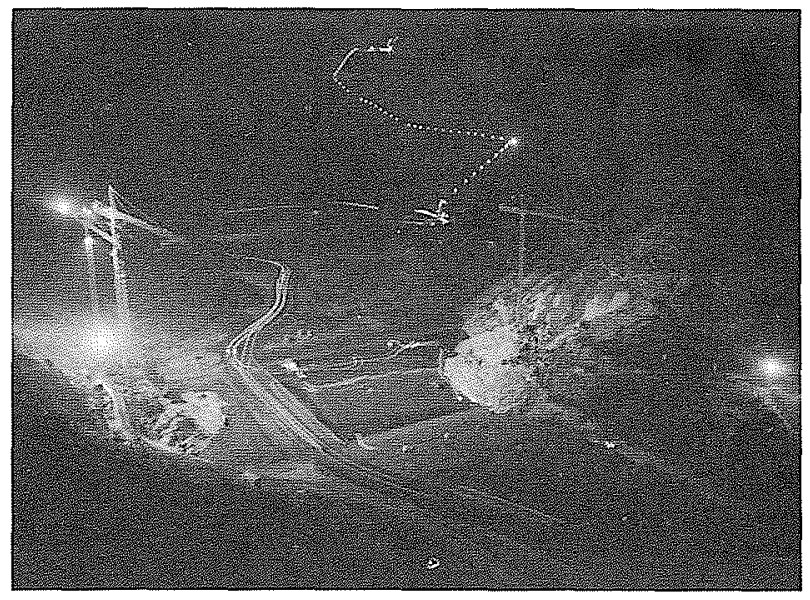

Figure 14 - Le chantier du barrage de Grand'Maison, de nuit (photo MIRABEL).

\section{Le barrage du Verney (Fig. 15)}

Le bassin du Verney est créé par un barrage en alluvions à masque amont en béton bitumineux. Le masque est raccordé à une paroi moulée en béton plastique pénétrant dans les alluvions sur $50 \mathrm{~m}$ de hauteur.
- Hauteur au-dessus du terrain naturel $=42 \mathrm{~m}$

- Longueur en crête $\quad=430 \mathrm{~m}$

- Largeur en crête $\quad=10 \mathrm{~m}$

- Pente moyenne du talus amont $=1 / 2$

- Pente moyenne du talus aval $=1 / 1,8$

- Volume du remblai $=1,5 \mathrm{hm}^{3}$

En rive gauche du barrage du Verney, une usine abritant un groupe de restitution de $11 \mathrm{MW}$, l'usine d'Oz, complète l'armement de la chute.

\section{Le circuit en charge}

1. Galerie en charge :

Longue de $7100 \mathrm{~m}$, elle se développe dans le cristallin de Belledonne, roche à caractéristiques particulièrement élevées, très dure et abrasive. Le diamètre de foration est de $7,70 \mathrm{~m}$. L'épaisseur du revêtement est de $0,30 \mathrm{~m}$ en terrain normal et $0,40 \mathrm{~m}$ dans les sections renforcées. Après appel d'offres, le choix s'est porté sur la foration mécanique, à l'aide d'un tunnelier Robbins (Fig. 17). La fenêtre principale, implantée à la Villette, dessert une attaque amont et une attaque aval.

\section{Cheminée d'équilibre}

Elle consiste en un puits de $10 \mathrm{~m}$ de diamètre et $200 \mathrm{~m}$ de hauteur entièrement blindé, muni d'un diaphragme, et une chambre d'expansion supérieure.

3. Conduites forcées

Les 12 groupes sont alimentés par trois conduites de $3,00 \mathrm{~m}$ de diamètre intérieur, bloquées au rocher, dans trois puits inclinés de $3,60 \mathrm{~m}$ de diamètre et de $1500 \mathrm{~m}$ de longueur, forés à $56 \%$ de pente par des tunneliers Wirth, modèle TB II.

Un $4^{\grave{e}}$ puits sert à l'accès hivernal et au drainage du rocher.

\section{Les usines de l'Eau d'Olle}

Les machines sont réparties dans deux usines implantées à la verticale l'une de l'autre :

- Usine souterraine, équipée de 8 groupes réversibles à quatre étages, de $150 \mathrm{MW}$ de puissance unitaire, $600 \mathrm{tr} / \mathrm{min}$;

- Usine de surface, équipée de 4 groupes Pelton à 5 jets, également de $150 \mathrm{MW}, 426 \mathrm{tr} / \mathrm{min}$. Outre leur aptitude au réglage fin de la puissance, ces groupes servent au démarrage dos à dos des pompes.

\section{Usine réversible}

Elle est creusée dans un lias ardoisier compact, à un niveau fixé par un enfoncement de $40 \mathrm{~m}$ de la roue inférieure sous le plan d'eau minimal. Elle comporte trois cavernes parallèles renfermant les robinets amont, les groupes et les batardeaux aval. La caverne des groupes a une hauteur totale de $39 \mathrm{~m}$, une largeur de $15 \mathrm{~m}$, une longueur de $162 \mathrm{~m}$. Elle est reliée à la surface par trois puits d'accès, quatre puits de transfert de puissance, 2 puits d'exhaure et de ventillation et une galerie en hélice à $10 \%$ de pente.

\section{Usine Pelton}

Elle est intégrée au talus naturel et a comme dimensions : $125 \mathrm{~m}$ de longueur et $28 \mathrm{~m}$ de largeur. Les locaux d'exploitation lui sont accolés. 


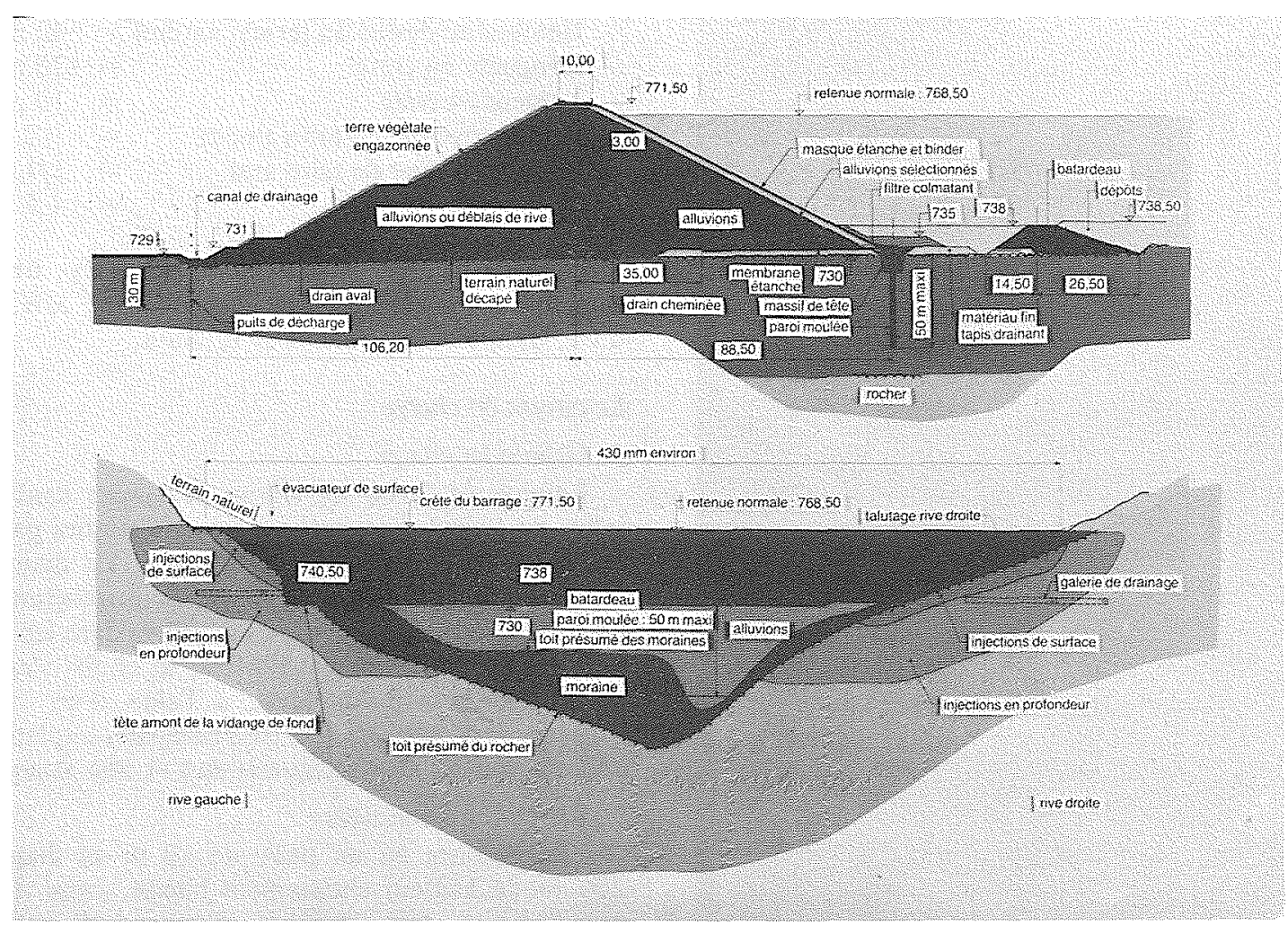

Figure 15 - Barrage du Verney - Coupes transversale et longitudinale.

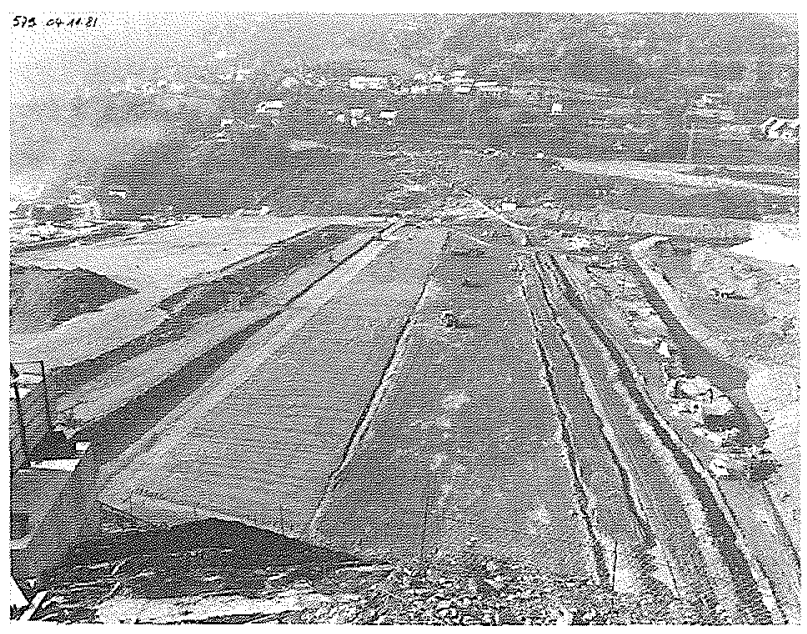

Figure 16 - Le chantier du barrage de Verney (photo PICCARDY).

Le volume total des excavations souterraines atteint $105000 \mathrm{~m}^{3}$, celui des bétons des deux usines $60000 \mathrm{~m}^{3}$.

\section{Programme des travaux}

La phase des travaux préparatoires, qui a commencé en 1978, a comporté notamment :

- Le remodelage des routes d'accès à la cuvette de Grand'Maison et à la fenêtre d'attaque de la galerie en charge,

- La dérivation provisoire de l'Eau d'Olle et le déblaie-

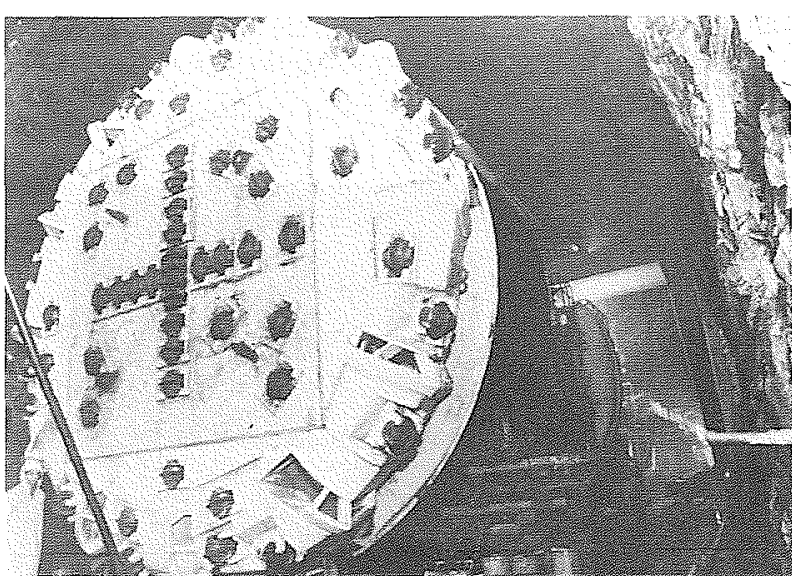

Figure 17 - Tête du tunnelier ROBBINS $\phi 7,70$ mètres.

ment des éboulis de pente dans l'emprise du barrage de Grand'Maison,

- La mise en place des infrastructures de chantier et d'accueil des entreprises.

Les principaux marchés ont été notifiés et les travaux correspondants engagés en 1980.

La situation actuelle du chantier est la suivante :

\section{Barrage de Grand'Maison}

Son programme d'exécution tient compte des condi- 


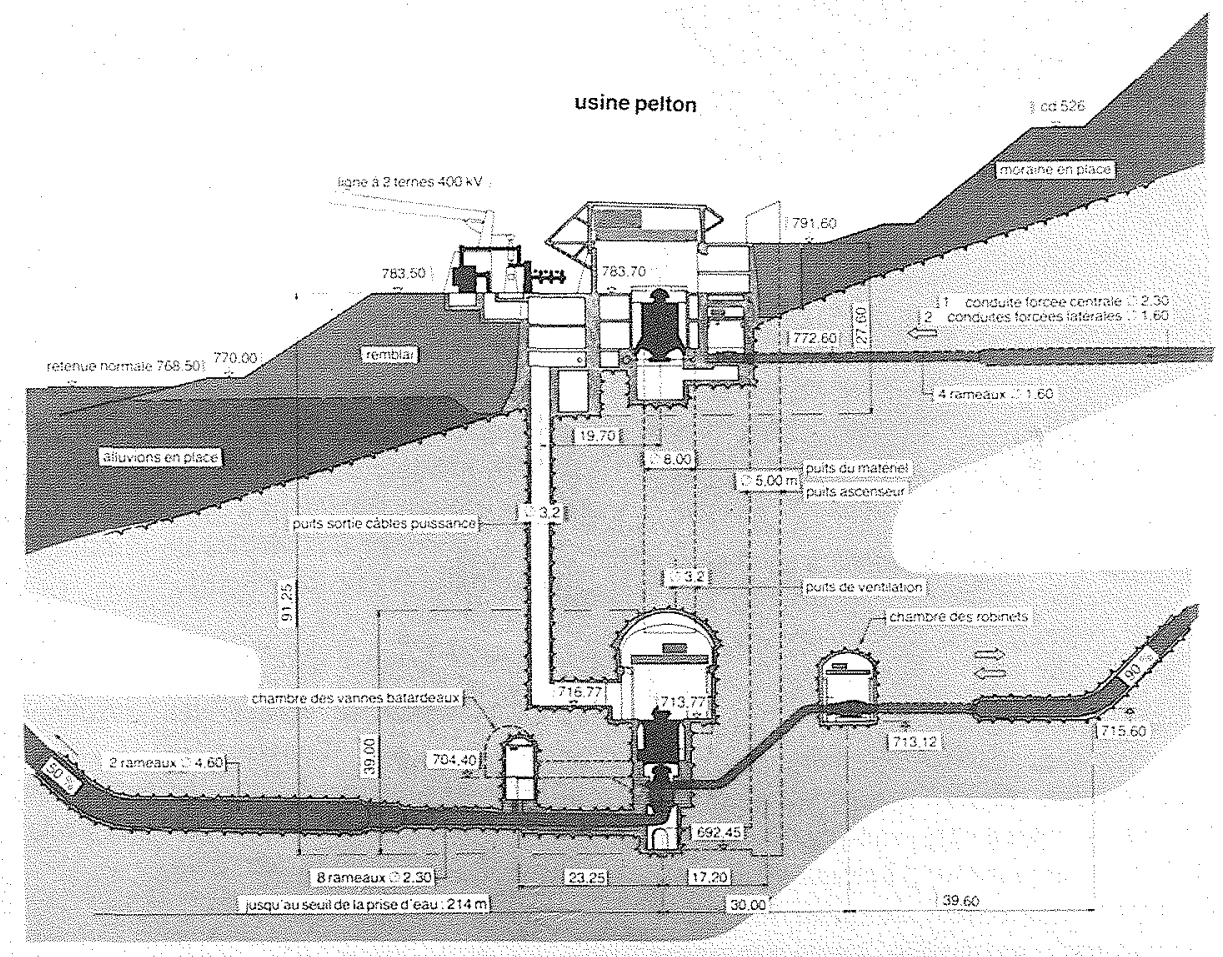

Figure 18 - Usines de l'Eau d'Olle - Coupe transversale.

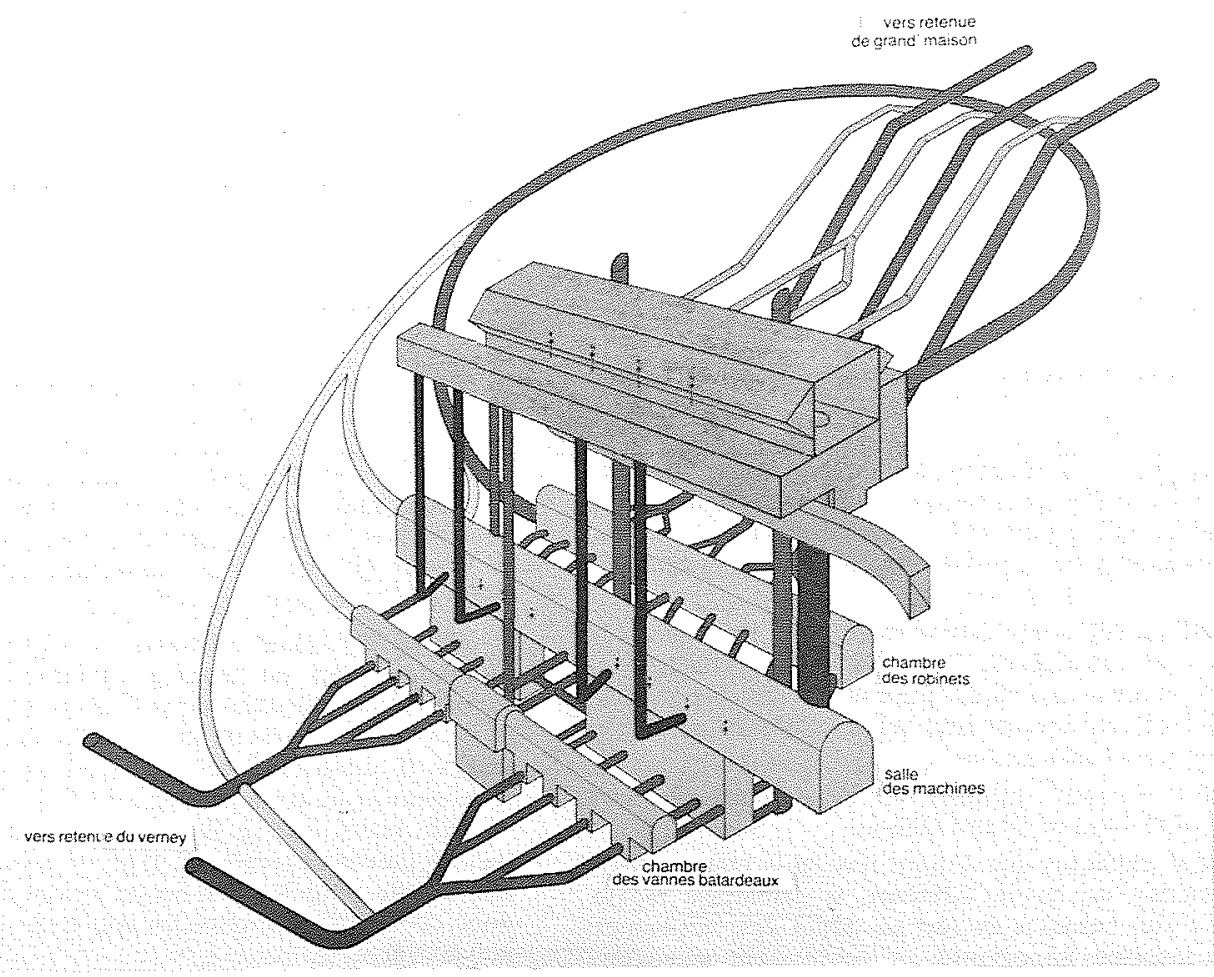

Figure 19 - Usines de l'Eau d'Olle - Schéma hydraulique. 


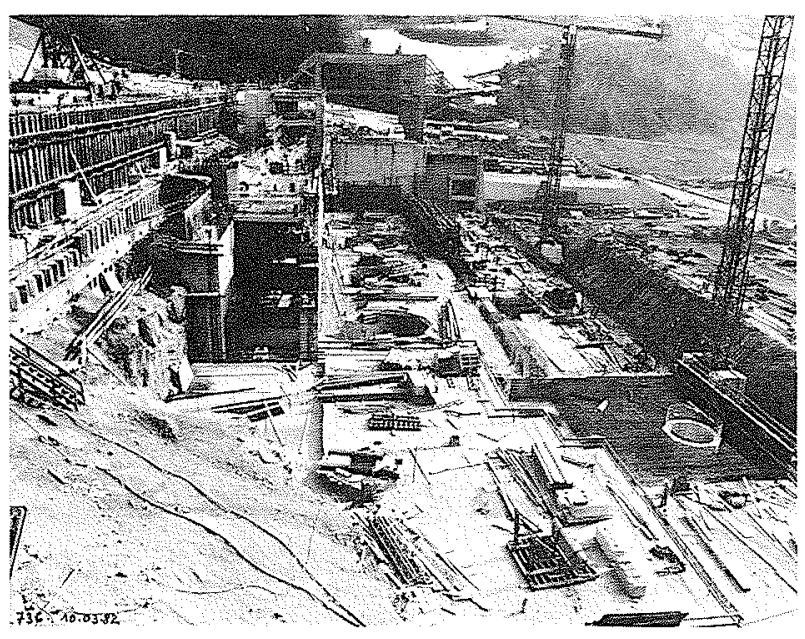

Figure 20 - Le chantier des usines de l'Eau d'Olle (photo PICCARDY)

tions géographiques qui sont particulièrement sévères : l'altitude et l'enneigement tardif n'autorisent qu'un chantier saisonnier et la durée des campagnes est en moyenne de 5 mois $1 / 2$ seulement ; l'exiguité du site, la raideur des rives et l'emplacement des gites, imposent des schémas de circulation compliqués. Après une phase de traitement de la fondation dans l'emprise du noyau, les remblais ont commencé de façon significative en 1981 et, au cours de cette campagne, $1,5 \mathrm{Mm}^{3}$ de maté. riaux ont été mis en place.

\section{Barrage du Verney}

Ce barrage constitue le chantier de repli hivernal du Groupement d'Entreprise chargé des terrassements. A l'heure actuelle, la paroi moulée est terminée et $600000 \mathrm{~m}^{3}$ de remblais sont en place.

\section{Galerie en charge}

La fenêtre principale, dans la vallée de Vaujany, a été exécutée à l'explosif. L'avancement actuel du tunnelier sur l'attaque amont est de $900 \mathrm{~m}$.

\section{Conduites forcées}

Le puits incliné de service et les 2 premiers puits de conduites forcées ont été forés à l'aide de tunneliers Wirth, modèle TB II, dans de bonnes conditions (avancement moyen supérieur à $200 \mathrm{~m}$ par mois).

Le montage des viroles de conduites forcées dans les puits inclinés est commencé.

\section{Usines de l'Eau d'Olle}

L'avancement actuel des dérochements de l'usine souterraine est d'environ $80 \%$. En ce qui concerne l'usine Pelton, les bétons de masse sont coulés et l'exécution des bétons de structure et de la charpente métallique se poursuit, en parallèle.

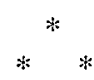

La première mise en eau du barrage de Grand'Maison est prévue pour l'été 1984. La mise en service des groupes s'étalera de fin 1984 à fin 1985.

\section{Discussion}

Président : M. DEVOISSELLE

M. le Président. - Je remercie M. COURIER qui vient de montrer d'une façon remarquable que le pompage hydraulique sera indispensable pour maintenir la qualité du réseau électrique, tant que la puissance des centrales nucléaires ne pourra pas être modulée.

M. I. de BEAUREGARD estime que, dans les calculs de rentabilité pour les aménagements hydroélectriques la comparaison avec les équipements thermiques de référence est pénalisante car l'hydroélectricité est une source d'origine nationale, renouvelable, propre et silencieuse.

M. MEGNINT demande comment ont été dimensionnées les turbines Pelton de l'usine de Grand'Maison.

$M$. COURIER explique que l'usine réversible étant dimensionnée en fonction du bassin inférieur, le démarrage rapide des pompes par des turbines Pelton en dos à dos, nécessitait une puissance installée importante en équipement gravitaire.
Des considérations supplémentaires de téléréglage et de souplesse de fonctionnement ont amené à choisir une puissance Pelton de $600 \mathrm{MW}$, ce qui a conduit à une puissance installée totale de $1800 \mathrm{MW}$ et à une puissance moyenne disponible de $1500 \mathrm{MW}$ seulement.

M. STURLESSE aimerait savoir quelles sont les possibilités actuelles de stockage d'énergie par volant d'inertie. Les systèmes existants actuellement sont de petite taille. Des projets plus importants avec volant de $100 \mathrm{t}$ sont à l'étude. Malgré la complexité de ces dispositifs de conversion d'énergie, le rendement pourrait atteindre $75 \%$.

Cependant, bien qu'il représente une source d'énergie décentralisée non négligeable, ce type de stockage a une autonomie insuffisante pour les besoins du réseau français ; il ne pourrait donc servir qu'à atténuer les appels de puissance pour certains industriels. 


\section{Abstract}

\section{Share of energy transfer by pumping in EDF's park of generating facilities and development of Grand'Maison}

Part I: The Economic approach to energy transfer

The paper explains the economic value of pumping, first by recalling the characteristics of demand for electricity, i.e. its daily, weekly, monthly and random variations. It also recalls the characteristics of thermal generation facilities and their cost of production.

It then spells out the "direct" method for optimizing the park of generating facilities and shows the resulting availability of low-load hours.

Energy transfer by pumping can then be justitied: comparison of pumping with competing systems, methodology of pumping efficiency studies, economic value of the different pumping modes: seasonal, weekly or daily.

Part I ends with the presentation of the current and projected programme relating to pumping.

Part 2: The Grand'Maison development
Grand'Maison is a twin development with a $1200 \mathrm{MW}$ plant, equipped with reversible turbines and a $600 \mathrm{MW}$ plant equipped with Pelton turbines, making it the most powerful hydro-electric development in France.

The upper reservoir capacity: $132 \mathrm{~h} \mathrm{~m}^{3}$ is formed by a rockfill dam with a vertical earth core; its height is $160 \mathrm{~m}$ and volume $12,5 \mathrm{hm}^{3}$. The lower reservoir is enclosed by a $42 \mathrm{~m}$ high alluvium dyke, sealed upstream.

The two reservoirs are separated by a maximum head of $955 \mathrm{~m}$. The circuit comprises a $7 \mathrm{~km}$ gallery bored by a tunnelling machine at a diameter of $7.70 \mathrm{~m}$ and three forced flow conduits installed in tilted shafts of length, $1500 \mathrm{~m}$ and diameter, $3.60 \mathrm{~m}$.

A $160 \mathrm{~m}$ long underground cavity houses the eight $150 \mathrm{MW}$ reversible sets. Above it is the Pelton plant, with four sets of identical power (150 MW)

The planned commissioning dates of this development are between end 1984 and end 1985 . 